\title{
Ethnobotanical knowledge on non-conventional food and medicinal plants in Rio Cajari Extractivist Reserve, Amazon, Brazil
}

Galdino Xavier de Paula Filho ( $\sim$ galdinoxpf@gmail.com )

Universidade Federal do Amapa https://orcid.org/0000-0002-0235-2072

Adivair Freitas Ribeiro

Universidade Federal do Amapa

Alcidete Flexa Moraes

Universidade Federal do Amapa

Willis Freitas Penha

Universidade Federal do Amapa

Wardsson Lustrino Borges

EMBRAPA Centro de Pesquisa Agroflorestal do Amapa

Ricardo Henrique Silva Santos

Universidade Federal de Vicosa

\section{Research}

Keywords: Indigenous knowledge, Folk medicine, Food security, Traditional populations, Amazon rainforest

Posted Date: September 3rd, 2020

DOl: https://doi.org/10.21203/rs.3.rs-35316/v2

License: @ (i) This work is licensed under a Creative Commons Attribution 4.0 International License. Read Full License 


\section{Abstract}

Background: Information on the knowledge, management and ways of using food and medicinal plants by traditional populations, family farmers and Brazilian native population in the Amazon is essential to guarantee the sovereignty of these groups. The objective of this study was to evaluate the diversity, knowledge and ways of using non-conventional food and medicinal plants in traditional communities in a protected area in the Brazilian Amazon.

Methods: This study was conducted using semi-structured interviews applied to local respondents. Fifty-six residents were interviewed in 26 communities. The Indices of Use Value (UVI) and relative frequency of species citation (Fr) were evaluated; also, their diversity and equitability using the Shannon- Wiener $\left(\mathrm{H}^{\prime}\right)$ Pielou $\left(\mathrm{J}^{\prime}\right)$ indices, respectively. The species were listed according to their family, scientific name, popular names, categories of use, propagation environment, growth habit, medicinal indications, domestication status, production cycle, and herbarium registration.

Results: A total of 269 species of both non-conventional food and medicinal plants were identified, distributed in 84 botanical families, 198 genera, in addition to 13 unidentified species. The Arecaceae and Lamiaceae families had the highest species richness ( 11 and 7 , respectively). Eryngium foetidum L. (Apiaceae) and Ipomoea batatas L. (Convolvulaceae) presented the highest relative citation frequencies (19.7 and 19.3, respectively) and the highest index of use value of the species (0.94 and 0.92, respectively). The ShannonWiener $\left(\mathrm{H}^{\prime}\right)$ and Pielou $\left(\mathrm{J}^{\prime}\right)$ diversity indices were considered high (5.02 and 0.9 , respectively).

Conclusions: It was observed that the studied species are consumed daily by the population of this protected area. In the environment in which these families are found, of geographical isolation and distance from urban centers, these species become the only food and medicinal resources, therefore, being fundamental to the sovereignty of these families.

\section{Background}

Brazil is a country of immense biodiversity, distributed throughout the biomes that occupy its territory. Among them, Amazonia stands out as the largest and the most preserved Brazilian biome, besides being the largest biodiversity reserve on the planet, occupying $49.3 \%$ of the national territory [1]. Its mega-biodiversity is currently estimated at around 2,500 tree species (representing one third of all tropical wood on the planet) and 30 thousand species of herbaceous plants and shrubs (out of a total of 100 thousand existing all over South America) [2]. This biome has a set of nature protected areas that are protected by law, such as the Extractive Reserves, where traditional populations, indigenous groups, quilombolas, riverside dwellers and family farmers live. These populations establish their forms of survival in line with and dependence on the available natural resources, especially non-conventional food plants and medicinal plants $[3,4]$.It is a region of low demographic density, cut by countless rivers and lakes $[5,6]$, with an enormous coverage of tropical forest that, although has suffered an intense process of deforestation and forest degradation in the last decades, still preserves an extensive area of native forest [7]. This region is inhabited by populations in need of infrastructure and health and education services. The population has a low level of education besides being located in isolated places with difficult access and far from urban centers $[8,9]$, where people find in the forest resources, especially non-conventional and medicinal food plants, the main food and medicinal resources, respectively $[10,11]$.

The non-conventional food plants are plant species widely used as food by populations living in rural Brazil [12]. They are species that, in many cases, spread spontaneously, have not undergone the process of agricultural technification and genetic improvement, as in the case of other agricultural cultures, however they have a broad relationship with the food security of many families that consume them daily [13]. These species have one or more parts that are used as human food and can be consumed directly, or providing oils, spices and condiments used in cooking [14]. While medicinal plants are plant species distributed in the environment and have metabolites in their structure, with chemical properties capable of combating and preventing the action of pathogens (fungi, bacteria, protozoa, viruses) harmful to the human organism [15]. It is noteworthy that there are no plant species only for food use and others used only for medicinal purposes. The vegetables have multiple purposes. There are cases where the same species is used in human food; in the elaboration of home remedies; for building homes, boats, tools and other utensils [16].

Considering these specificities, these populations have developed a set of skills and tacit knowledge about the ways of using these forest resources over the years, adapting the survival strategies of the social groups living in these regions, whose knowledge has been tried, validated and transmitted through generations [17]. However, in the course of the last few years, due to an accelerated process of exploration of these areas, this knowledge has been lost [18], so it is necessary and urgent to carry out studies that investigate the 
potential of these plant species, which are associated with strategies for sovereignty, food security and therapeutically of these population groups $[10,19]$.

In aspect of ethnobiology, the present study is interesting mainly because of the multiplicity of uses that the investigated population performs on plant species, as well as the tacit knowledge developed and expanded on these species that enabled the development of forms of preparation, use, management and cultivation by several generations. The present study, although apparently similar to others carried out in the Amazon, stands out for having been developed with traditional populations living in protected areas, far from urban centers; however pressured by forest concession projects (Jari Celulose Project), mineral (RENCA - National Copper and Associates Reserve) and road (BR 156) projects, both strong components of the historical disorderly occupation process in the Amazon. This study can contribute to the understanding of the relationship of these plant species with the sovereignty and food security of dozens of families that depend on them.

This study investigated the diversity, knowledge and ways of using non-conventional food plants and medicinal plants by traditional populations (collectors, family farmers, agro-collectors, quilombolas, indigenous and riverside inhabitants) in the Cajari River Extractive Reserve, state of Amapá, in the Brazilian Amazon. This protected area is located on the left bank of the Amazon River, an area cut by dozens of rivers and lakes, rich in plant biodiversity, fishing resources and wild animals that constitute the food base of th0e population residing in this protected area.

\section{Methods}

Study sites

The present study aimed to investigate the diversity, knowledge and ways of using non-conventional food plants and medicinal plants by

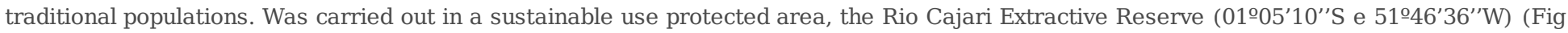
1), which has an area of 532,397.20 hectares, and is located on the left bank and delta of the Amazon River, in the south of the State of Amapá. This unit is protected by law. It was created by Presidential Decree No. 99.145, of March 12, 1990 [20]. Its predominant vegetation is terra firme forests (the highest part of the unit), tidal flooded forests (intermediate part of the unit where floods occur and ebb from rivers) and flooded fields (next to the curves of rivers and streams, as well as rivers, streams and lakes.

The Cajari River Extractive Reserve covers the territory of three municipalities: Mazagão, Vitória do Jari and Laranjal do Jari, and has a population of 4,164 inhabitants [21]. It is located between the Maracá Agroextractive Settlement Project, at the BR 156 Highway (Macapá-Jari), the Jari Celulose Project and the Amazon River. Its population consists predominantly of family farmers who develop agroextractive activities (migratory farming of slash and burn agriculture, collection of fruits and vegetables in the forest and artisanal fishing); quilombolas whose inhabitants descended from slaves who came to the municipality of Mazagão in the late $19^{\text {th }}$ century; indigenous people of the Waiãpi ethnic group, riverside dwellers and extractivists who have the natural resources as their main source of survival.

The study was concentrated along three of the main rivers that make up the protected area, exactly where the communities where the collectors reside, in which the rivers Cajari, Muriacá and Amazonas (left bank) are located. In the Extractive Reserve there are communities, groups of several residences that are formed in strategic regions of the rivers, usually close to an inlet; in a place close to the mainland, favorable to small farms; with a vast abundance of certain products (Brazil nuts, Acai), fishing resources; or even in strategic locations for navigation and local commerce, such as the mouth of rivers and igarapes.

The houses of the families are built of wood collected in the forest, covered with wood chips or asbestos tiles. The houses are built high above the level of the river and igarapes to avoid flooding during the Amazonian winter (rainy season from February to June). In most communities there is a level-I elementary school (up to the fifth grade), and in all communities there is the presence of religious institutions 
(Catholicism predominates, but with a marked presence of evangelical churches), elements of African and indigenous religions are also found there, showing strong religious syncretism.

\section{Field trips and authorization for execution of the study}

Four trips were made to the study area. Each trip lasted 12 to 15 days, from December 2016 to March 2017. These trips to the study area consisted of establishing an experience process with the community to conduct interviews and participate in their daily activities as well as the usage relationships with food and medicinal plants [22].

During the home visits, we sought to identify people recognized as owners of the ethnobotanical knowledge about medicinal and food plants [23]. To reach them, we counted with the help of key interviewed, such as agents of the unit's management body, leaders of local organizations, teachers of the unit's schools and some students from the Federal University of Amapá (UNIFAP) who are from RESEX Rio Cajari [24].

All the participants of this study were informed of its objective. They all agreed to participate and signed the Free and Informed Consent Form provided by the Ethics Committee in Research with Human Beings of the Federal University of Viçosa (CEP/ UFV), via Brazil Platform (Opinion number: 1.718.017).

Permissions for the study were obtained from the following agencies:

The research was registered in the National Genetic Heritage Management System (SisGen), which establishes criteria to access the genetic heritage and associated traditional knowledge, in accordance with the requirements of the biodiversity law (access registration number: A4DCD0D) [25].

The "Authorization for activities with a scientific purpose" was requested and obtained from the Biodiversity Authorization and Information System (SISBIO), an agency linked to the Ministry of the Environment (MMA), in order to obtain permission for the collection and transportation of biological material (authorization number: 55801-1).

The access to the protected area depended on authorization from the unit's managing body, the Chico Mendes Institute for Biodiversity Conservation (ICMBio), which issued the respective authorization, as well as the access to communities and their respective research interviewed were communicated and authorized by the residents' associations.

\section{Ethnobotanical data collection}

Ethnobotanical information was collected through interviews with local experts who were appointed by the key-interviewed. Were considered key-interviewed the people residing in the communities, recognized as references in knowledge about the use and benefits of plants (food and medicinal), those generally consulted by other members of the communities about indication and use of the new plants. In the interviews, semi-structured questionnaires containing pre-defined topics were applied, allowing the rise of new questions during the dialogue and the interviewed could spontaneously express their ideas about the use of plants for food and medicinal purposes [26]. 
Before starting the interviews, a pre-test of the interview script was carried out, with a group of five interviewed, in order to assess the clear understanding and precision of the terms, unfolding and order of questions, in addition to other information.

\section{Plant species collection and identification}

The collections of plant species were performed in vivo, with the participation of the interviewee, using the technique known as "guided tour" [27], in order to obtain the identification and more accurate information about the indicated species, following the methodological standards defined for ethnobotanical studies [28].

The species were registered by means of photographs and on records for the collection of botanical material.

Triplicates of each species were collected, then identified by means of comparison with samples from the Herbarium Collection of Amapá State (HAMAB), specialized bibliographies $[12,15]$. Subsequently, the exsiccates were herborized and incorporated into the collection of HAMAB, a faithful depository of samples of components of the Genetic Heritage of the Amazon, in the State of Amapá.

\section{Data analysis}

The homogeneity and diversity of food and medicinal species were assessed using the Shannon-Wiener Biological Diversity Index (H') and the Pielou Equitability Index (J') $[29,30]$. These indices are used to assess the species richness in the studied area and the distribution of knowledge about plants among the research interviewed, respectively, were calculated for all food and medicinal plant species found in the present study, using the following equations:

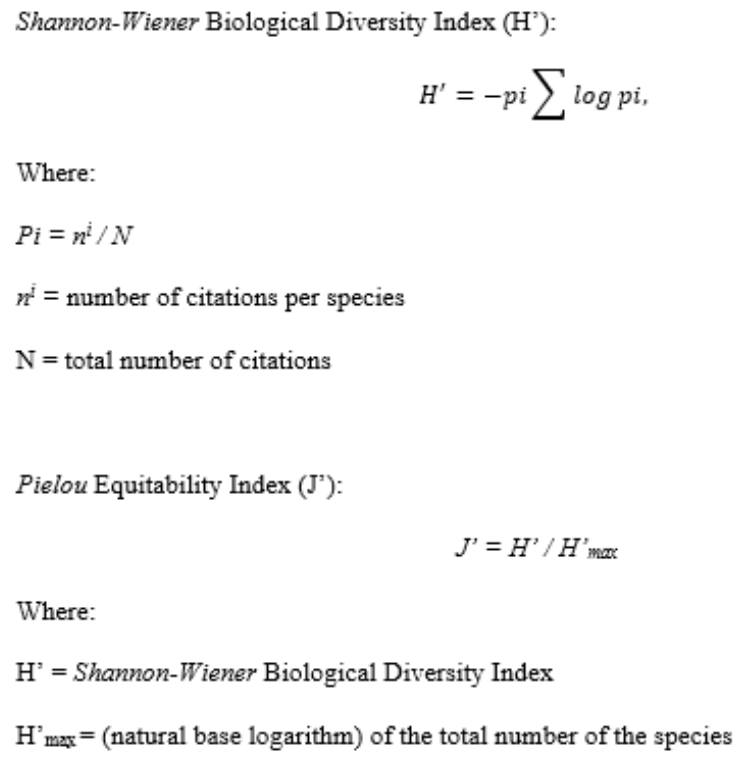$$
H^{\prime}=-p i \sum \log p i
$$

Where:

$P i=n^{i} / N$

$n^{i}=$ number of citations per species

$\mathrm{N}=$ total number of citations

Pielou Equitability Index ( $\left.\mathrm{J}^{\prime}\right)$ :

$$
J^{\prime}=H^{\prime} / H^{\prime}{ }_{\max }
$$

Where:

$\mathrm{H}^{\prime}=$ Shannon-Wiener Biological Diversity Index

$\mathrm{H}^{\prime}{ }_{\max }=$ (natural base logarithm) of the total number of the species

These indexes were compared with the indexes of similar studies carried out in the Brazilian Amazon and also in other regions, inside and outside Brazil. 
The daily demand of species for food and medicinal use was evaluated, using the Use Value Index (IVU) [31]. This index is used to assess how well the species are known and used by the local population [32]. The IVU was obtained using the following equation:

$$
I V U=\sum U / n
$$

Where:

$\mathrm{U}=$ Number of citations of the species

$\mathrm{n}=$ Total number of research interviewed

The Relative Citation Frequency $(\mathrm{Fr})$ indicates how much particular species stands out in relation to the

rest of the others, and expresses how well the species is known [29]. This index was obtained using the

following equation:

$$
F r=\sum(U x 100) / N
$$

$\mathrm{U}=$ Number of citations of the species

$\mathrm{N}=$ Number of species found in the study

The species were classified as non-conventional food, medicinal and dual-purpose (food and medicinal) [33]. The propagation environment (vegetable garden, orchard, forest, family farm and riparian forest) and growth habit (creeping, climbing, herbaceous, shrub and tree) of the species were observed and categorized [34], in addition to the forms of use and therapeutic indications in the case of medicinal plants [35].

A classification was made regarding the domestication status of these species in order to verify whether they are cultivated, or whether they propagate spontaneously [34], as well as their production cycles in annual, semi-perennial or perennial.

\section{Results And Discussion}

\section{Social-cultural characteristics}

The information was obtained from 56 interviewed, residing in 26 communities along these three rivers, which totaled 2,896 citations of food and medicinal vegetable species, are shown in Table 1.

The Tapereira quilombola community stood out with the largest number of interviewed, as it has historically accumulated an inheritance in the use of plant resources based on the knowledge inherited from their African ancestors [33]. The Tapereira community resulted in the highest number of species citations. However, the best citation average was generated in the Santana community as the interviewed in this community are familiar with a greater number of species of food and medicinal plants. The fact that the Tapereira and Santana communities had the highest number and average number of plant species has contributed to the fact that the Cajari River had the highest number of plant species mentioned (1215) and the average number of visited rivers (52.8 plant species / interviewee) (Table 1).

Table 1. Rivers, communities, respondents (resp.), citations on food and medicinal plants in the Cajari River Extractive Reserve, Amazon, Brazil. 


\begin{tabular}{|c|c|c|c|c|c|c|c|}
\hline \multirow[t]{2}{*}{ River } & \multirow[t]{2}{*}{ Communities } & \multirow[t]{2}{*}{ Resp. / community } & \multirow[t]{2}{*}{ Resp. / river } & \multirow[t]{2}{*}{ Citations / community } & \multirow[t]{2}{*}{ Citations / river } & \multicolumn{2}{|c|}{ Average of citations } \\
\hline & & & & & & Community & River \\
\hline \multirow[t]{4}{*}{ Muriacá } & Aterro do Muriacá & 3 & \multirow[t]{8}{*}{16} & 170 & \multirow[t]{8}{*}{803} & 56,7 & \multirow[t]{8}{*}{50,2} \\
\hline & Boa Vista & 2 & & 89 & & 44,5 & \\
\hline & Comércio & 1 & & 44 & & 44 & \\
\hline & Conceição do Muriacá & 6 & & 329 & & 54,8 & \\
\hline \multirow[t]{4}{*}{8 communities } & Mirituba & 1 & & 35 & & 35 & \\
\hline & Santa Helena & 1 & & 42 & & 42 & \\
\hline & São Luis & 1 & & 45 & & 45 & \\
\hline & Vila Nova & 1 & & 49 & & 49 & \\
\hline \multirow{9}{*}{9 communities } & Costureira & 1 & \multirow[t]{9}{*}{23} & 39 & \multirow[t]{9}{*}{1215} & 39 & \multirow[t]{9}{*}{52,8} \\
\hline & Formigueiro & 2 & & 97 & & 48,5 & \\
\hline & Paraíso & 2 & & 127 & & 63,5 & \\
\hline & Poção & 2 & & 76 & & 38 & \\
\hline & Santa Rita & 3 & & 144 & & 48 & \\
\hline & São Sebastião & 3 & & 141 & & 47 & \\
\hline & Tapereira & 7 & & 357 & & 51 & \\
\hline & Terra Vermelha & 1 & & 66 & & 66 & \\
\hline & Vila Santana & 2 & & 168 & & 84 & \\
\hline \multirow{10}{*}{9 communities } & Foz do Rio Ajuruxi & 4 & \multirow[t]{9}{*}{17} & 268 & \multirow[t]{9}{*}{878} & 67 & \multirow[t]{9}{*}{51,6} \\
\hline & Rio Ariramba & 1 & & 38 & & 38 & \\
\hline & Rio Arirambinha & 2 & & 76 & & 38 & \\
\hline & Rio Bispo & 1 & & 53 & & 53 & \\
\hline & Rio Capitão & 2 & & 136 & & 68 & \\
\hline & Rio Carneiro & 1 & & 24 & & 24 & \\
\hline & Rio Chato & 1 & & 58 & & 58 & \\
\hline & Rio Mulato & 2 & & 96 & & 48 & \\
\hline & Vila Betel & 3 & & 129 & & 43 & \\
\hline & & 56 & 56 & 2896 & 2896 & & \\
\hline
\end{tabular}

The methodological procedure adopted in the present study resulted in the finding of 56 plant specialists (37 women and 19 men), aged between 25 and 97 years old, and with the following social occupations: farmers, extractivists, artisans, builders, fishermen, chestnut collectors, rubber tappers, carpenters, shamans and midwives.

According to the interviewed, the ethnobotanical knowledge about PANC (Non-Conventional Food Plants) and medicinal plants is concentrated in adults. Of the 56 interviewed, 31 are people aged between 25 and 59 years old, whose average age is $58.9 \pm 14.6$. Although there are many elderly people, these results differ from other studies found in the literature in which they show that ethnobotanical knowledge about PANC and medicinal plants is concentraded in the elderly [34, 36, 37]. It is worth mentioning that the average age in the state of Amapá is 73.9 years [38], which is $20 \%$ higher than the average age of the interviewed in this study, thus, showing that this population, even though younger, has their ways of lives more dependent on the local plant resources.

The interviewees admitted that the young population of the unit are still interested in food and medicinal resources, although it differs from other studies on the subject [39], denominated "cultural erosion" by some authors [40]. However, it was possible to observe through the reports by the interviewed that in recent years, the phenomenon of urbanization in rural areas (access to traditionally urban goods and services, change in the income profile of some families), associated with the scarcity of some food resources (fish, and plants) has impacted the ways of life of local populations, gradually arousing greater interest in other food sources, and medicines in the pharmaceutical industry.

The relationship between the forms of use of these species occurs mainly because they are found in the same environment, propagated or cultivated using the same techniques. This relationship was established, above all, due to the geographic isolation in which these families are found, far from urban centers and without the possibility of income so to acquire other sources of food and medicines, therefore, these species have established themselves as the main resources, whose relationship is observed in other parts of Brazil and the world [41, 42]. 


\section{Non-conventional food and medicinal plants}

A total of 269 plant species used for food and medicinal purposes were identified. They were distributed in 84 families and 198 botanical genera, resulting in a total of 2,896 citations. The species with the highest number of citations were E. foetidum (Apiaceae) and $I$. batatas (Convolvulaceae), which were cited 53 and 52 times, respectively. E foetidum is a species of medicinal and food use. For therapeutic purposes, the tea boiled from its roots is indicated to fight parasites of the human organism, and as a food use, its leaves are cooked together with other foods. I. batatas is used for food purposes only, its tubers are cooked and served for breakfast (Table 2).

Table 2 contains information about the species identified in this study. The botanical families Arecaeae and Lamiaceae had the largest number of food and medicinal species, 19 and 17 species, respectively, with a predominance of species used for medicinal purposes. A situation also observed in other studies carried out with traditional populations in the Amazon, including studies carried out in rural communities in the municipality of Manacapuru, in Amazonas, Brazil [43]. In other communities in this municipality (Manacapuru/AM, Brazil), Costa, Mitja analyzed plant resources used by family farmers and observed a predominance of resources used for medicinal purposes [44].

In relation to the propagation environment of the species, it was found in the forest, vegetable gardens, orchards, Family farms and riparian forests (Table 2), but with predominance for those found in the forest (120 species) and in the vegetable gardens (65 species). This situation occurs mainly for two reasons. Firstly, because the study region is an environmental protected area in which plant extractions is the main source of income of the families, hence their strong relationship with the forest, as observed by Silva, Fantini, Shanley [45]. Secondly, because the communities where the families live are floodplain areas, which are flooded throughout the year, and like their residences, the gardens are also built with wood, or planted in canoes suspended from the ground, to prevent flooding and attack by animals that are raised loose, like buffalo. This form of cultivation in suspended beds has already been recorded in other studies with traditional populations and farmers in the Brazilian Amazon [43, 44].

Vegetable gardens are places where families grow herbaceous species, built on a wooden structure, in which the soil, fertilizer and pots with the plants are placed; are located behind residences and elevated from the ground to protect against flooding rivers and animals. Orchards resemble agroforestry backyards, are located around residences, are forests (medicinal, food and other uses) formed by randomly established tree species, with no defined spacing. Family farms are openings held annually in the middle of the forest, through cutting and burning of vegetation, in these places the species of annual cycle are cultivated, used by the family for food and medicinal purposes.Also, it is observed that the relationship between propagation environment, growth habit, stage of domestication and vegetative cycles is associated with the physiological characteristics of plants since there is no way to establish a tree species in a suspended garden, nor to cultivate a herbaceous in a shaded forest environment as observed in a study carried out on the use and knowledge of plants by traditional populations of the Tapajós National Forest (Santarém PA / Brazil) [17]. The availability of these plant resources is associated with the social organization of families, since herbaceous species are required on a daily basis, and for this reason they are found in vegetable gardens and orchards, being easily accessible for women who deal with household activities [46]. The species located in the forest are seasonal, which makes them to be obtained, making them less required [46].

According to the interviewed' report, the number of PANC species and medicinal plants is decreasing and are found with more difficulties, mainly forest species with wood and food value, such as Endopleura uchi (Huber) Cuatrec, Caryocar villosum (Aubl.) Pers., Bertholletia excelsa H.B.K., Tabebuia roseoalba (Ridl.) Sandwith. This situation has occurred mainly due to the raise in the local population, which demanded the construction of more gardens and, consequently, increased the pressure on the river and the forest, but also due to the outbreaks of burning and deforestation that has frequently occurred within the unit. This is an aggravating factor, since many species are 
directly related to the food security of these families and there is no agronomic protocol, with propagation and management techniques that make it possible to replant seedlings.

The availability and seasonality of the species influence the social organization of families and communities [47]. This situation was observed in this study, since the forest provides many fruit food species, however they are only available during the rainy season (February to June). After this period, the main food species are grown in the family farms (particularlry the rhizomes and some herbaceous) during the Amazonian summer (July to December). The gardens are perennial and have food and medicinal species throughout the year. Medicinal resources such as leaves, seeds and bark are also available year-round in forests, riparian forests and orchards.

It was found in this study 138 plant species for food use. Of these, 96 are fruit species (the others are vegetables, seeds and rhizomes), and correspond to $69.5 \%$ of the food species found in here. Vegetables are consumed with food and represent only $6 \%$ of this percentage, which corroborates the fact that fruits are more present in the diet of traditional populations in the Amazon, as already observed in other studies [19, 44], together with cassava flour (Manihot esculenta Crantz.) and fish [48, 49]. Also, the consumption of vegetables is low among this population group, as highlighted by Adams, Murrieta, Sanches [50].

Among the species found in the study and available in Table 2, it was observed that some of them, from the families Acanthaceae and Amaranthaceae, has the same popular name of the trade name of some medicines sold by the pharmaceutical industry, such as the anador species (Justicia pectoralisvar. stenophylla Leonard), melhoral (Justicia pectoralis Jacq.), ampicilina (Alternanthera tenella Colla), penicilina (Gomphrena arborescens L.f.), terramicina (Alternanthera brasiliana (L.) Kuntze), cibalena (Artemisia vulgaris L.), elixir paregórico (Ocimum selloi Benth.), insulina (Cissus sicyoides L.), vique grande (Mentha spicata L.) and vique pequeno (Mentha arvensis L.). Other authors have already found similar to the one in which these home remedies are associated with names of industrialized medicines, usually prepared in the form of teas [51]. A possible explanation for the attribution of the name of industrialized remedies to many medicinal plants may be related to the influence of allopathic medicine in rural areas, in which the name given to these plants has something to do with the smell, taste or effect of an industrialized medicine [51, 52].

Regarding the non-conventionality of food vegetable species, it is observed that in regions with more technified agriculture, some PANC species are already duly improved genetically and established in commercial cultivation systems, as well Anacardium occidentale, Mangifera indica, Cocos nucifera, Aloe vera, Ruta graveolens, Citrus sinensis, among others. However, authors like Kinupp and Lorenzi; Leal and collaborators emphasize that the condition of non-conventionality is established by the relationship between the plant species and the populations that use it, and also by the technical conditions (cultivation and genetic improvement) in which the species is found [12, 14]. These conditions corroborate what is observed in ethnobotanical studies, which express above all, the relationship and importance of these species for the food security strategies of families residing in regions where these species are the only food resource [14].

Table 2. Botanical family, scientific and popular name, category of use, propagation environment, growth habit, forms of use, medicinal indications, domestication stage and registration of food and medicinal plant species found in the Cajari River Extractive Reserve, Amazon, Brazil. 


\begin{tabular}{|c|c|c|c|c|c|c|c|c|c|}
\hline Botanical family & Scientific name & Popular name & Cat. & Env. & Grow. & Forms of use & $\begin{array}{c}\text { Medicinal } \\
\text { indications }\end{array}$ & Dom. & Reg. \\
\hline \multirow[t]{3}{*}{ Acanthaceae } & $\begin{array}{l}\text { Justicia pectoralisvar. } \\
\text { stenophylla Leonard }\end{array}$ & Anador & M & $\mathrm{Vg}$ & $\mathrm{He}$ & Leaf tea & $\begin{array}{l}\text { Headache and } \\
\text { stomachache }\end{array}$ & $\mathrm{C}$ & $\begin{array}{l}\text { INPA } \\
20639 \\
\end{array}$ \\
\hline & Justicia pectoralis Jacq. & Melhoral & $\mathrm{M}$ & $\mathrm{Vg}$ & $\mathrm{He}$ & Leaf tea & Headache & $\mathrm{C}$ & $\begin{array}{c}\text { INPA } \\
106185 \\
\end{array}$ \\
\hline & $\begin{array}{l}\text { Justicia acuminatissima } \\
\text { (Miq.) Bremek }\end{array}$ & Saratudo & $\mathrm{M}$ & $\begin{array}{l}\mathrm{Vg} \\
\mathrm{O}\end{array}$ & $\mathrm{He}$ & Leaf tea & $\begin{array}{c}\text { Tranquilizer, } \\
\text { fever and } \\
\text { measles }\end{array}$ & C, S & $\begin{array}{c}\text { INPA } \\
223272\end{array}$ \\
\hline Adoxaceae & $\begin{array}{l}\text { Sambucus australis } \\
\text { Cham. \& Schltdl. }\end{array}$ & Sabugueiro & $\mathrm{M}$ & $\begin{array}{l}\mathrm{Vg}, \\
\mathrm{O}\end{array}$ & $\mathrm{He}$ & Leaf tea & Inflammation & C, S & $\begin{array}{c}\text { INPA } \\
208304\end{array}$ \\
\hline \multirow[t]{4}{*}{ Amaranthaceae } & $\begin{array}{l}\text { Alternanthera tenella } \\
\text { Colla }\end{array}$ & $\begin{array}{l}\text { Ampicilina de } \\
\text { planta }\end{array}$ & M & $\mathrm{Vg}$ & $\mathrm{He}$ & $\begin{array}{l}\text { Leaf tea with } C \text {. } \\
\text { spicatus, } P \text {. } \\
\text { niruri and } A \text {. } \\
\text { muricata leaves }\end{array}$ & $\begin{array}{l}\text { Stomachache; } \\
\text { urinary tract } \\
\text { infection }\end{array}$ & $\mathrm{C}$ & $\begin{array}{c}\text { INPA } \\
71449\end{array}$ \\
\hline & $\begin{array}{l}\text { Chenopodium } \\
\text { ambrosioides L. }\end{array}$ & Mastruz & M & $\begin{array}{c}\mathrm{Vg} \\
\mathrm{O}\end{array}$ & $\mathrm{He}$ & $\begin{array}{l}\text { Leaf tea with } E \text {. } \\
\text { foetidum and } C \text {. } \\
\text { papaya roots }\end{array}$ & Worm & C, S & $\begin{array}{c}\text { INPA } \\
277507\end{array}$ \\
\hline & $\begin{array}{l}\text { Gomphrena arborescens } \\
\text { L.f. }\end{array}$ & Penicilina & $\mathrm{M}$ & $\mathrm{Vg}$ & $\mathrm{He}$ & $\begin{array}{l}\text { Leaf tea with } C \text {. } \\
\text { spicatus, } P \text {. } \\
\text { niruri and } A \text {. } \\
\text { muricata leaves }\end{array}$ & $\begin{array}{l}\text { Urinary tract } \\
\text { infection }\end{array}$ & $\mathrm{C}$ & $\begin{array}{r}\text { INPA } \\
81315\end{array}$ \\
\hline & $\begin{array}{l}\text { Alternanthera brasiliana } \\
\text { (L.) Kuntze }\end{array}$ & Terramicina & $\mathrm{M}$ & $\mathrm{Vg}$ & $\mathrm{He}$ & Leaf tea & Headache & $\mathrm{C}$ & $\begin{array}{c}\text { INPA } \\
220460 \\
\end{array}$ \\
\hline \multirow[t]{6}{*}{ Anacardiaceae } & $\begin{array}{l}\text { Anacardium occidentale } \\
\text { L. }\end{array}$ & Caju & Mf & $\mathrm{O}$ & $\operatorname{Tr}$ & $\begin{array}{l}\text { M: Tree bark } \\
\text { tea - F: fresh } \\
\text { fruit }\end{array}$ & Diarrhea & S & $\begin{array}{l}\text { CEN } \\
65224 \\
\end{array}$ \\
\hline & Anacardium giganteum L. & Caju açu & Mf & Fo & $\operatorname{Tr}$ & $\begin{array}{c}\text { M: Tree bark } \\
\text { tea - F: fresh } \\
\text { fruit } \\
\end{array}$ & Diarrhea & S & $\begin{array}{l}\text { INPA } \\
61226\end{array}$ \\
\hline & Curatella americana L. & Caju do mato & $\mathrm{F}$ & Fo & $\operatorname{Tr}$ & Fresh fruit & & S & $\begin{array}{c}\text { HAMAB } \\
9415\end{array}$ \\
\hline & $\begin{array}{l}\text { Schinus terebinthifolia } \\
\text { Raddi }\end{array}$ & Ceru & Mf & Fo & $\operatorname{Tr}$ & $\begin{array}{l}\text { M: Tree bark } \\
\text { tea - F: almond }\end{array}$ & Gastritis; worm & S & $\begin{array}{r}\text { INPA } \\
139911 \\
\end{array}$ \\
\hline & Mangifera indica L. & Manga & Mf & $\mathrm{O}$ & $\operatorname{Tr}$ & $\begin{array}{c}\text { M: Tree bark } \\
\text { tea; bath: leaves } \\
\text { of } M \text {. } \\
\text { paradisíaca, } E \text {. } \\
\text { oleracea, } C \text {. } \\
\text { nucifera and } C \text {. } \\
\text { citratus / F: } \\
\text { fresh fruit and } \\
\text { juice } \\
\end{array}$ & $\begin{array}{l}\text { Stomachache; } \\
\text { bathe woman } \\
\text { after childbirth }\end{array}$ & S & $\begin{array}{c}\text { INPA } \\
262837\end{array}$ \\
\hline & Spondias mombin L. & $\begin{array}{l}\text { Taperebá } \\
\text { (cajá) }\end{array}$ & Mf & Fo & $\operatorname{Tr}$ & $\begin{array}{l}\text { M: grind the } \\
\text { tree knot and } \\
\text { spread over the } \\
\text { wound / F: fresh } \\
\text { fruit and juice }\end{array}$ & Healing & $\mathrm{S}$ & $\begin{array}{c}\text { INPA } \\
141180\end{array}$ \\
\hline \multirow[t]{4}{*}{ Annonaceae } & Annona glabra L. & Araticum & F & Fo & $\operatorname{Tr}$ & Fresh fruit & & $\mathrm{S}$ & $\begin{array}{c}\text { INPA } \\
270299 \\
\end{array}$ \\
\hline & Annona mucosa Jacq. & Biribá & Mf & Fo & $\operatorname{Tr}$ & $\begin{array}{c}\text { M: put tree bark } \\
\text { it in the water } \\
\text { until it gets } \\
\text { colored and } \\
\text { drink it / F: } \\
\text { fresh fruit and } \\
\text { juice } \\
\end{array}$ & Sore throat & $\mathrm{C}$ & $\begin{array}{l}\text { INPA } \\
2181\end{array}$ \\
\hline & Annona montana Macfad. & Conde & $\mathrm{F}$ & $\mathrm{O}$ & $\operatorname{Tr}$ & Fresh fruit & & S & $\begin{array}{c}\text { INPA } \\
246115 \\
\end{array}$ \\
\hline & Annona muricata L. & Graviola & Mf & $\mathrm{O}$ & $\operatorname{Tr}$ & $\begin{array}{c}\text { M: beverage of } \\
\text { the leaves with } \\
\text { A. esperanzae } \\
\text { problem; leaf } \\
\text { tea with } A \text {. } \\
\text { tenella leaves, } \\
P \text {. niruri and } C \text {. } \\
\text { spicatus / F: } \\
\text { fresh fruit and } \\
\text { juice } \\
\end{array}$ & $\begin{array}{l}\text { Aches over the } \\
\text { body; urinary } \\
\text { tract infection }\end{array}$ & S & $\begin{array}{l}\text { INPA } \\
75580\end{array}$ \\
\hline \multirow[t]{2}{*}{ Apiaceae } & $\begin{array}{l}\text { Arracacia xanthorriza } \\
\text { Bancr. }\end{array}$ & Batata crioula & $\mathrm{F}$ & Ff & $\mathrm{Cr}$ & Cooked rhizome & & $\mathrm{C}$ & BOTU \\
\hline & Eryngium foetidum L. & Chicória & Mf & $\mathrm{Vg}$ & $\mathrm{He}$ & M: tea of the & Worm & C, S & INPA \\
\hline
\end{tabular}




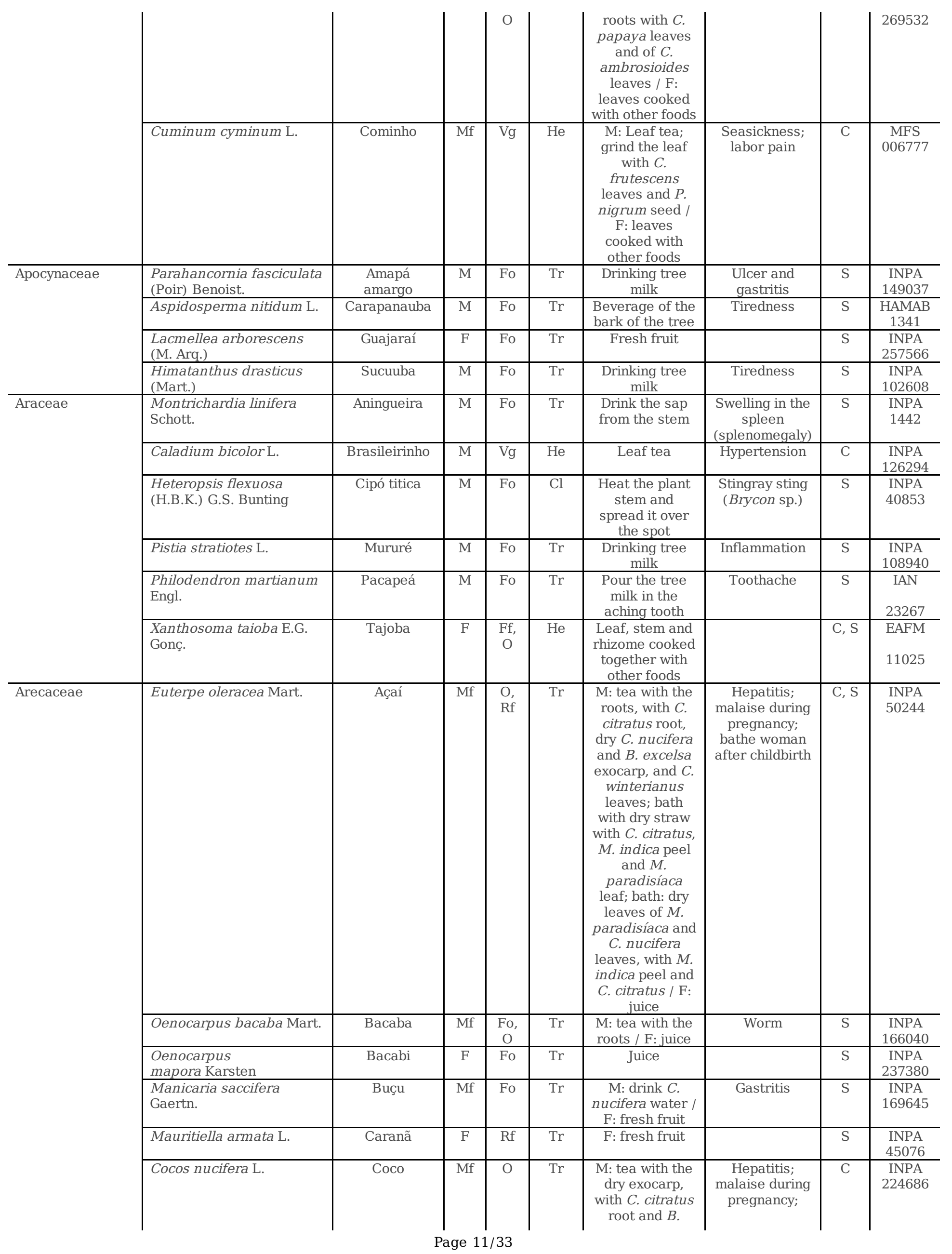




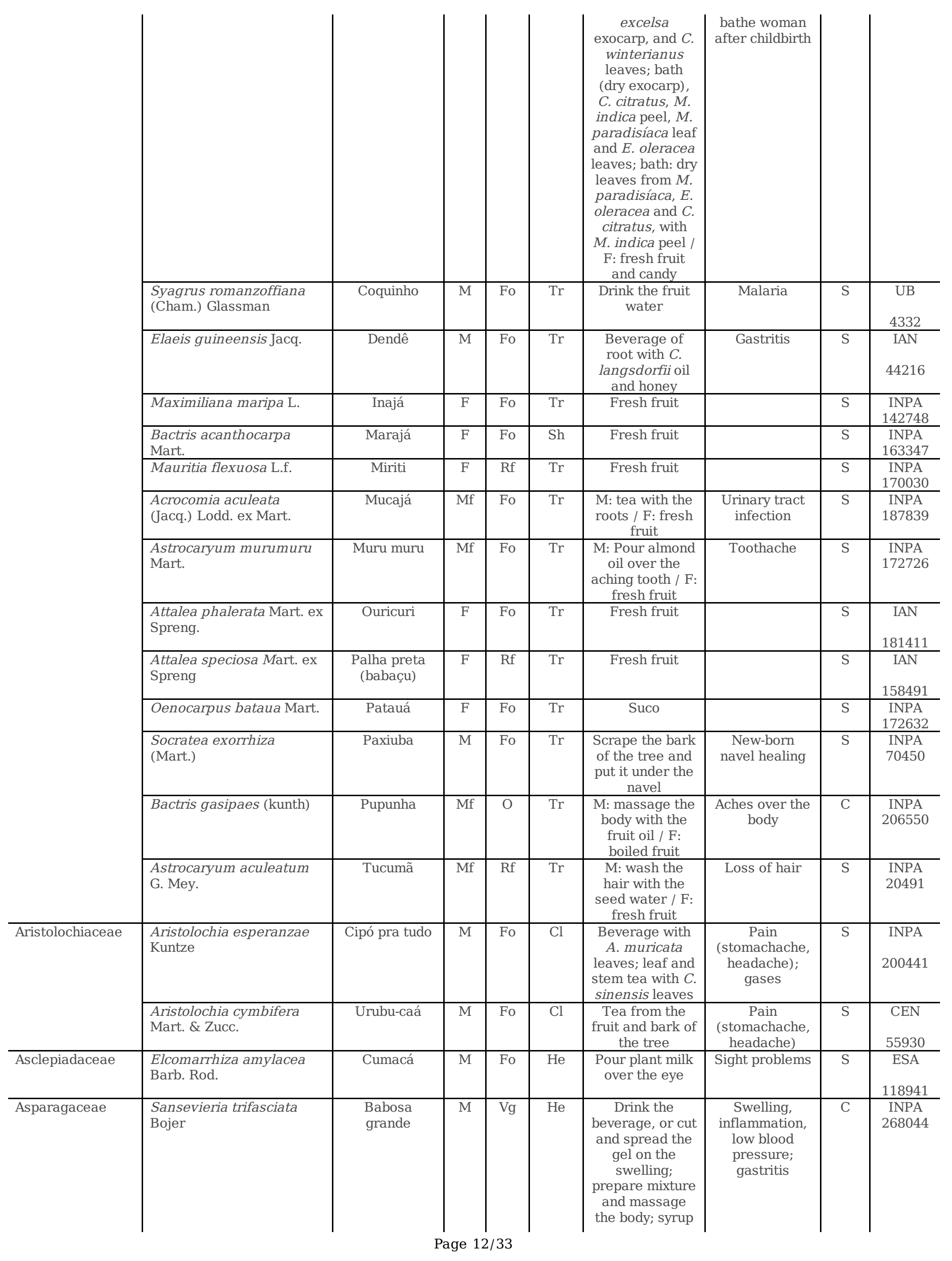




\begin{tabular}{|c|c|c|c|c|c|c|c|c|c|}
\hline & & & & & & $\begin{array}{c}\text { with } K . \\
\text { brasiliensis or } \\
\text { B. pinnatum } \\
\text { leaves and } \\
\text { honey } \\
\end{array}$ & & & \\
\hline Asphodelaceae & Aloe vera (L.) Burn. f. & $\begin{array}{c}\text { Babosa } \\
\text { pequena }\end{array}$ & $\mathrm{M}$ & $\mathrm{Vg}$ & $\mathrm{He}$ & $\begin{array}{c}\text { Drink the } \\
\text { beverage, or cut } \\
\text { and spread the } \\
\text { gel on the } \\
\text { swelling; } \\
\text { prepare mixture } \\
\text { and massage } \\
\text { the body; syrup } \\
\text { with } K \text {. } \\
\text { brasiliensis or } \\
\text { B. pinnatum } \\
\text { leaves and } \\
\text { honey } \\
\end{array}$ & $\begin{array}{l}\text { Swelling, } \\
\text { inflammation, } \\
\text { low blood } \\
\text { pressure; } \\
\text { gastritis }\end{array}$ & $\mathrm{C}$ & $\begin{array}{c}\text { INPA } \\
106256\end{array}$ \\
\hline \multirow[t]{12}{*}{ Asteraceae } & $\begin{array}{l}\text { Gymnanthemum } \\
\text { amygdalinum (Delie) }\end{array}$ & Boldo africano & $\mathrm{M}$ & $\mathrm{Vg}$ & $\mathrm{He}$ & $\begin{array}{l}\text { Leaf tea, with } \\
\text { C. rotundus and } \\
\text { F. chica leaves }\end{array}$ & $\begin{array}{c}\text { Malaria, } \\
\text { diabetes and } \\
\text { cirrhosis }\end{array}$ & $\mathrm{C}$ & $\begin{array}{c}\text { IAN } \\
192804\end{array}$ \\
\hline & Matricaria recutita L. & Camomila & $\mathrm{M}$ & $\mathrm{Vg}$ & $\mathrm{He}$ & Leaf tea & Tranquilizer & $\mathrm{C}$ & UB \\
\hline & & & & & & & & & 25796 \\
\hline & Tanacetum vulgare L. & $\begin{array}{l}\text { Catinga de } \\
\text { mulata }\end{array}$ & $\mathrm{M}$ & $\mathrm{Vg}$ & $\mathrm{He}$ & $\begin{array}{l}\text { Leaf tea. Leaf } \\
\text { mixture with } \\
\text { alcohol, leaves } \\
\text { from } R \text {. } \\
\text { officinalis, } R \text {. } \\
\text { graveolens and } \\
\text { S. orientale } \\
\text { seed } \\
\end{array}$ & $\begin{array}{l}\text { Headache; } \\
\text { diarrhea, } \\
\text { stroke }\end{array}$ & $\mathrm{C}$ & $\begin{array}{l}\text { EAFM } \\
11721\end{array}$ \\
\hline & Artemisia vulgaris L. & Cibalena & $\mathrm{M}$ & $\mathrm{Vg}$ & $\mathrm{He}$ & Leaf tea & $\begin{array}{c}\text { cramps, } \\
\text { hemorrhage } \\
\text { and fever }\end{array}$ & $\mathrm{C}$ & $\begin{array}{c}\text { INPA } \\
195375\end{array}$ \\
\hline & $\begin{array}{l}\text { Mikania cordifolia (L.f.) } \\
\text { Willd. }\end{array}$ & Cipó sucuriju & $\mathrm{M}$ & Fo & $\mathrm{Sh}$ & $\begin{array}{l}\text { Beverage of the } \\
\text { bark of the vine }\end{array}$ & Gastritis & $\mathrm{S}$ & $\begin{array}{l}\text { INPA } \\
11390 \\
\end{array}$ \\
\hline & Tagetes minuta L. & $\begin{array}{c}\text { Cravo de } \\
\text { planta }\end{array}$ & $\mathrm{M}$ & $\mathrm{Vg}$ & $\mathrm{He}$ & Leaf bath & Flu and cold & $\mathrm{C}$ & $\begin{array}{c}\text { INPA } \\
208085 \\
\end{array}$ \\
\hline & $\begin{array}{l}\text { Clibadium surinamense } \\
\text { Linn. }\end{array}$ & Cunambi & $\mathrm{M}$ & Ff & $\mathrm{He}$ & Seed tea & Pneumonia & $\mathrm{C}$ & $\begin{array}{l}\text { INPA } \\
7304 \\
\end{array}$ \\
\hline & $\begin{array}{l}\text { Acmella oleracea (L.) R.K. } \\
\text { Jansen }\end{array}$ & Jambu & Mf & $\mathrm{Vg}$ & $\mathrm{He}$ & $\begin{array}{l}\text { M: beverage of } \\
\text { the leaves with } \\
\text { bee honey and } \\
\text { C. guianensis } \\
\text { oil / F: leaf } \\
\text { cooked with } \\
\text { other foods }\end{array}$ & $\begin{array}{l}\text { Sore throat } \\
\text { and flu }\end{array}$ & $\mathrm{C}$ & $\begin{array}{c}\text { INPA } \\
234141\end{array}$ \\
\hline & $\begin{array}{l}\text { Eupatorium ayapana } \\
\text { Vent. }\end{array}$ & Japana branca & $\mathrm{M}$ & $\mathrm{Vg}$ & $\mathrm{He}$ & Leaf tea & Headache & $\mathrm{C}$ & $\begin{array}{c}\mathrm{JPB} \\
40991\end{array}$ \\
\hline & $\begin{array}{l}\text { Eupatorium triplinerve } \\
\text { Vahl. }\end{array}$ & Japana roxa & $\mathrm{M}$ & $\mathrm{Vg}$ & $\mathrm{He}$ & Leaf tea & Headache & $\mathrm{C}$ & $\begin{array}{c}\text { IAN } \\
182445 \\
\end{array}$ \\
\hline & Chaptalia nutans (L.) Pol. & $\begin{array}{l}\text { Língua de } \\
\text { vaca }\end{array}$ & $\mathrm{M}$ & $\mathrm{O}$ & $\mathrm{He}$ & Leaf tea & Hemorrhage & $\mathrm{S}$ & $\begin{array}{r}\text { INPA } \\
139919 \\
\end{array}$ \\
\hline Basellaceae & Basella alba L. & $\begin{array}{c}\text { Couve } \\
\text { manteiga }\end{array}$ & $\mathrm{F}$ & $\mathrm{Vg}$ & $\mathrm{He}$ & $\begin{array}{l}\text { Sautéed with } \\
\text { other foods }\end{array}$ & & $\mathrm{C}$ & $\begin{array}{c}\text { INPA } \\
202567 \\
\end{array}$ \\
\hline \multirow[t]{5}{*}{ Bignoniaceae } & Tabebuia caraiba (Mart.) & Cariobeira & $\mathrm{M}$ & Fo & $\operatorname{Tr}$ & Tree bark tea & Inflammation & $\mathrm{S}$ & $\begin{array}{c}\text { INPA } \\
208861\end{array}$ \\
\hline & $\begin{array}{l}\text { Mansoa alliacea (Lam.) } \\
\text { A.H. Gentry }\end{array}$ & Cipó alho & Mf & Fo & $\mathrm{Cl}$ & $\begin{array}{c}\text { M: tea and } \\
\text { beverage of the } \\
\text { leaves / F: } \\
\text { cooked with } \\
\text { other foods } \\
\end{array}$ & $\begin{array}{l}\text { Aches over the } \\
\text { body }\end{array}$ & $\mathrm{S}$ & $\begin{array}{c}\text { INPA } \\
177754\end{array}$ \\
\hline & $\begin{array}{l}\text { Tanaecium } \\
\text { nocturnum (Barb. Rodr.) }\end{array}$ & Cipó curimbó & M & Fo & $\mathrm{Cl}$ & $\begin{array}{l}\text { Leaf and bark } \\
\text { tea }\end{array}$ & $\begin{array}{l}\text { Protect against } \\
\text { "evil eye" }\end{array}$ & $\mathrm{S}$ & $\begin{array}{c}\text { IAN } \\
134799 \\
\end{array}$ \\
\hline & Bignonia exoleta Vell. & $\begin{array}{c}\text { Cipó } \\
\text { morceguinho } \\
\text { (unha de } \\
\text { morcego) } \\
\end{array}$ & $\mathrm{M}$ & Fo & $\mathrm{Cl}$ & Stem tea & $\begin{array}{l}\text { Headache and } \\
\text { stomachache }\end{array}$ & $\mathrm{S}$ & $\begin{array}{c}\text { IAC } \\
25183\end{array}$ \\
\hline & Crescentia cujete L. & Cuia & $\mathrm{M}$ & $\mathrm{O}$ & $\operatorname{Tr}$ & $\begin{array}{c}\text { Leaf bath; bath } \\
\text { with bark of } \\
\text { nazarana tree }\end{array}$ & $\begin{array}{c}\text { Flu and cold; } \\
\text { fever and "evil } \\
\text { eye" }\end{array}$ & $\mathrm{S}$ & $\begin{array}{c}\text { INPA } \\
262772\end{array}$ \\
\hline
\end{tabular}




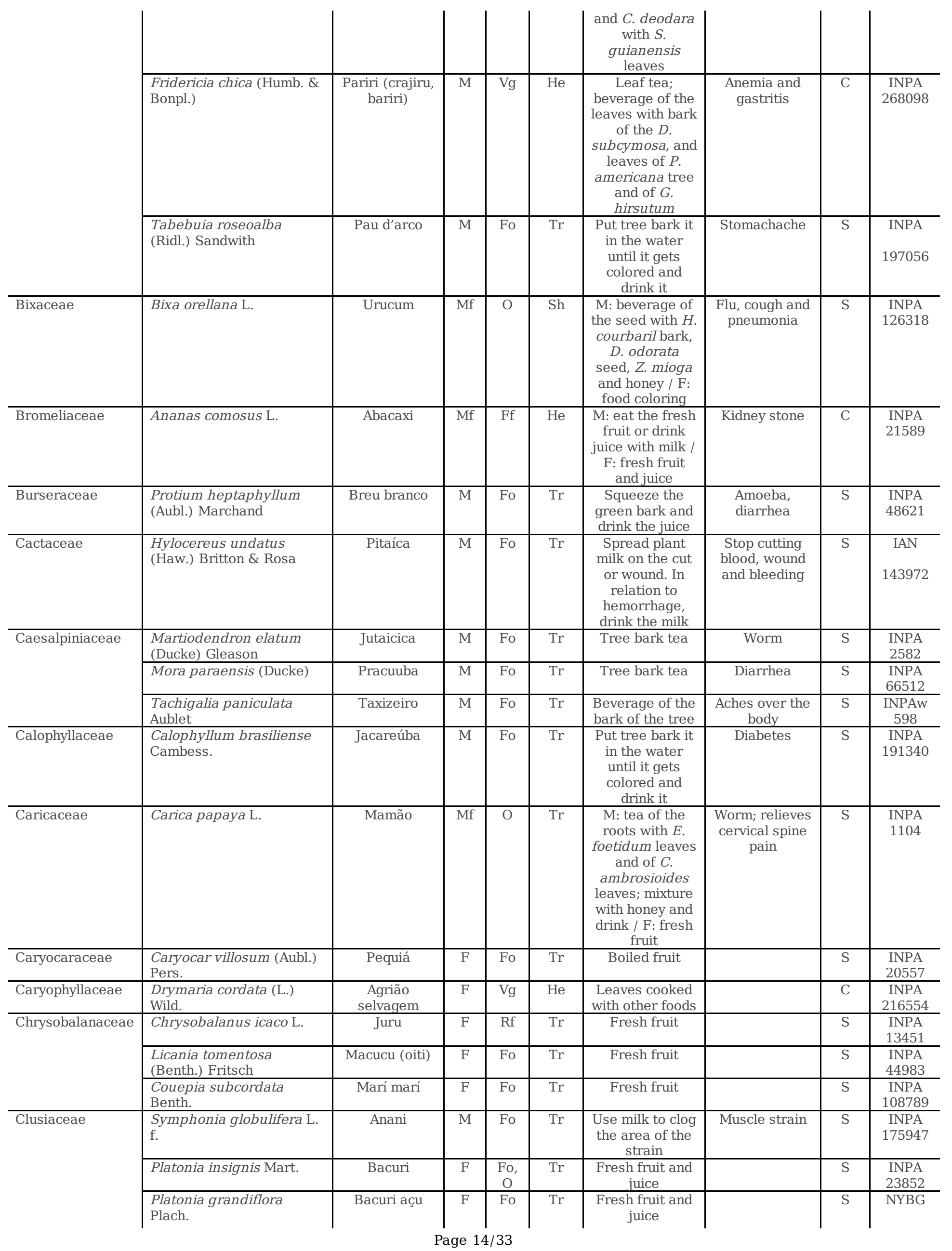




\begin{tabular}{|c|c|c|c|c|c|c|c|c|c|}
\hline & & & & & & & & & 2730667 \\
\hline & $\begin{array}{l}\text { Garcinia madruno (Kunth) } \\
\text { Hammel }\end{array}$ & Bacuri azedo & $\mathrm{F}$ & Fo & $\operatorname{Tr}$ & $\begin{array}{l}\text { Fresh fruit and } \\
\text { juice }\end{array}$ & & S & $\begin{array}{l}\text { INPA } \\
92041 \\
\end{array}$ \\
\hline & Garcinia brasiliensis Mart. & $\begin{array}{c}\text { Bacuri liso } \\
\text { (bacurizinho) }\end{array}$ & $\mathrm{F}$ & Fo & $\operatorname{Tr}$ & $\begin{array}{l}\text { Fresh fruit and } \\
\text { juice }\end{array}$ & & $\mathrm{S}$ & $\begin{array}{l}\text { INPA } \\
98907 \\
\end{array}$ \\
\hline & $\begin{array}{l}\text { Vismia guianensis (Aubl.) } \\
\text { Pers. }\end{array}$ & Lacre & $\mathrm{M}$ & Fo & $\operatorname{Tr}$ & $\begin{array}{l}\text { Squeeze the } \\
\text { juice from the } \\
\text { leaves on the } \\
\text { affected area }\end{array}$ & $\begin{array}{l}\text { Mycosis and } \\
\text { skin irritation }\end{array}$ & $\mathrm{S}$ & $\begin{array}{c}\text { INPA } \\
178820\end{array}$ \\
\hline \multirow[t]{3}{*}{ Convolvulaceaea } & Ipomoea batatas (L.) & Batata doce & $\mathrm{F}$ & $\begin{array}{c}\text { Ff, } \\
\text { O }\end{array}$ & $\mathrm{Cr}$ & Cooked & & C, S & $\begin{array}{l}\text { INPA } \\
3882\end{array}$ \\
\hline & $\begin{array}{l}\text { Ipomoea batatas (L.) var. } \\
\text { Rainha }\end{array}$ & Batata rainha & F & $\begin{array}{c}\text { Ff, } \\
\text { O }\end{array}$ & $\mathrm{Cr}$ & Cooked & & $\mathrm{C}, \mathrm{S}$ & $\begin{array}{c}\text { IAN } \\
169732\end{array}$ \\
\hline & $\begin{array}{l}\text { Ipomoea } \\
\text { purga (Wender.) Hayne }\end{array}$ & Batatão & $\mathrm{M}$ & Ff & $\mathrm{Cr}$ & $\begin{array}{l}\text { Grind the } \\
\text { rhizome, put it } \\
\text { in the water } \\
\text { until it gets } \\
\text { colored and } \\
\text { drink it }\end{array}$ & $\begin{array}{l}\text { Elimination of } \\
\text { toxic } \\
\text { substances in } \\
\text { the blood }\end{array}$ & $\mathrm{C}$ & $\begin{array}{c}\text { MBM } \\
214631\end{array}$ \\
\hline Costaceae & $\begin{array}{l}\text { Costus spicatus (Jacq.) } \\
\text { Sw. }\end{array}$ & Cana ficha & $\mathrm{M}$ & $\mathrm{O}$ & $\mathrm{Sh}$ & $\begin{array}{l}\text { Leaf and stem } \\
\text { tea with } A \text {. } \\
\text { tenella leaves, } \\
\text { of } P \text {. niruriand } \\
\text { of } A \text {. muricata; } \\
\text { beverage of } V \text {. } \\
\text { surinamensis } \\
\text { bark with } \\
\text { "mangangá" } M \text {. } \\
\text { acuminata }\end{array}$ & $\begin{array}{l}\text { Urinary tract } \\
\text { infection; } \\
\text { uterine } \\
\text { infection }\end{array}$ & $\mathrm{S}$ & $\begin{array}{c}\text { INPA } \\
268099\end{array}$ \\
\hline \multirow[t]{2}{*}{ Crassulaceae } & $\begin{array}{l}\text { Kalanchoe brasiliensis } \\
\text { Cambess. }\end{array}$ & $\begin{array}{l}\text { Pirarucu } \\
\text { branco (são } \\
\text { raimundo) }\end{array}$ & $\mathrm{M}$ & $\begin{array}{c}\text { VG, } \\
\mathrm{O}\end{array}$ & $\mathrm{He}$ & $\begin{array}{c}\text { Syrup: mixture } \\
\text { the leaves with } \\
\text { Aloe sp. and } \\
\text { honey; leaf tea }\end{array}$ & Gastritis & C, S & $\begin{array}{c}\text { IAN } \\
165540\end{array}$ \\
\hline & $\begin{array}{l}\text { Bryophyllum pinnatum } \\
\text { (Lam.) Oken }\end{array}$ & $\begin{array}{l}\text { Pirarucu roxo } \\
\text { (são } \\
\text { raimundo) }\end{array}$ & $\mathrm{M}$ & $\begin{array}{c}\mathrm{Vg} \\
\mathrm{O}\end{array}$ & $\mathrm{He}$ & $\begin{array}{l}\text { Syrup: mixture } \\
\text { the leaves with } \\
\text { Aloe sp. and } \\
\text { honey; leaf tea }\end{array}$ & Gastritis & C, S & $\begin{array}{c}\text { INPA } \\
268096\end{array}$ \\
\hline \multirow[t]{5}{*}{ Cucurbitaceae } & $\begin{array}{l}\text { Luffa operculata (L.) } \\
\text { Cogn. }\end{array}$ & $\begin{array}{c}\text { Buchinha } \\
\text { (cabacinha) }\end{array}$ & $\mathrm{M}$ & $\mathrm{O}$ & $\mathrm{Cl}$ & $\begin{array}{l}\text { Cut the fruit, } \\
\text { boil it in oil and } \\
\text { massage the } \\
\text { local; dried fruit } \\
\text { tea with the } \\
\text { root of the } P \text {. } \\
\text { angulata, and } \\
\text { leaves of } Q \text {. } \\
\text { amara and } A \text {. } \\
\text { grandifolia }\end{array}$ & $\begin{array}{l}\text { Hematoma; } \\
\text { malaria }\end{array}$ & $\mathrm{C}$ & $\begin{array}{c}\text { INPA } \\
224139\end{array}$ \\
\hline & Cucurbita pepo L. & $\begin{array}{l}\text { Jerimum } \\
\text { (abóbora) }\end{array}$ & Mf & $\begin{array}{c}\text { Ff, } \\
\mathrm{O}\end{array}$ & $\mathrm{Cr}$ & $\begin{array}{l}\text { M: stem tea / F: } \\
\text { fruit cooked } \\
\text { with other foods }\end{array}$ & Rheumatism & C, S & $\begin{array}{c}\text { INPA } \\
235420\end{array}$ \\
\hline & Cucumis anguria L. & Maxixe & $\mathrm{Mf}$ & $\begin{array}{c}\text { Ff, } \\
\text { O }\end{array}$ & $\mathrm{Cr}$ & $\begin{array}{c}\text { Fruit cooked } \\
\text { with other foods } \\
(\mathrm{M} / \mathrm{F})\end{array}$ & Cholesterol & C, S & $\begin{array}{l}\text { INPA } \\
1082\end{array}$ \\
\hline & Citrullus lanatus (Thunb.) & Melancia & Mf & $\begin{array}{c}\mathrm{Ff}, \\
\mathrm{O}\end{array}$ & $\mathrm{Cr}$ & $\begin{array}{l}\text { M: grind the } \\
\text { seed, put it in } \\
\text { the water and } \\
\text { drink it / F: } \\
\text { fresh fruit }\end{array}$ & Stroke & $\mathrm{C}, \mathrm{S}$ & $\begin{array}{l}\text { INPA } \\
56793\end{array}$ \\
\hline & $\begin{array}{l}\text { Sicana odorifera (Vell.) } \\
\text { Naudin }\end{array}$ & Melão caipira & $\mathrm{F}$ & $\begin{array}{c}\text { Ff, } \\
\mathrm{O}\end{array}$ & $\mathrm{Cl}$ & $\begin{array}{l}\text { Fresh fruit and } \\
\text { juice }\end{array}$ & & C, S & $\begin{array}{l}\text { INPA } \\
8973 \\
\end{array}$ \\
\hline \multirow[t]{2}{*}{ Cyperaceae } & Cyperus articulatus L. & Pripioca & $\mathrm{M}$ & $\mathrm{Vg}$ & $\mathrm{He}$ & $\begin{array}{c}\text { Grind the } \\
\text { rhizome, make } \\
\text { bath or tea and } \\
\text { massage the } \\
\text { body }\end{array}$ & $\begin{array}{l}\text { Aches over the } \\
\text { body }\end{array}$ & $\mathrm{C}$ & $\begin{array}{l}\text { INPA } \\
21149\end{array}$ \\
\hline & Cyperus rotundus L. & Tiririca & $\mathrm{M}$ & Fo & $\mathrm{He}$ & $\begin{array}{c}\text { Leaf tea, with } P \text {. } \\
\text { barbatus and } \\
\text { from } F \text {. chica } \\
\text { leaves }\end{array}$ & Diabetes & S & $\begin{array}{l}\text { INPA } \\
2202\end{array}$ \\
\hline \multirow[t]{3}{*}{ Dioscoreaceae } & $\begin{array}{l}\text { Dioscorea dodecaneura } \\
\text { Vell. }\end{array}$ & Cará branco & $\mathrm{F}$ & $\begin{array}{c}\text { Ff, } \\
\mathrm{O}\end{array}$ & $\mathrm{Cl}$ & Cooked rhizome & & C, S & $\begin{array}{c}\text { INPA } \\
192411\end{array}$ \\
\hline & Dioscorea bulbifera L. & Cará do ar & $\mathrm{F}$ & $\begin{array}{c}\text { Ff, } \\
\mathrm{O}\end{array}$ & $\mathrm{Cl}$ & Boiled fruit & & C, S & $\begin{array}{l}\text { MFS } \\
8146 \\
\end{array}$ \\
\hline & Dioscorea altissima Lam. & Cará mão de & $\mathrm{F}$ & Ff, & $\mathrm{Cl}$ & Cooked rhizome & & C, S & INPA \\
\hline
\end{tabular}




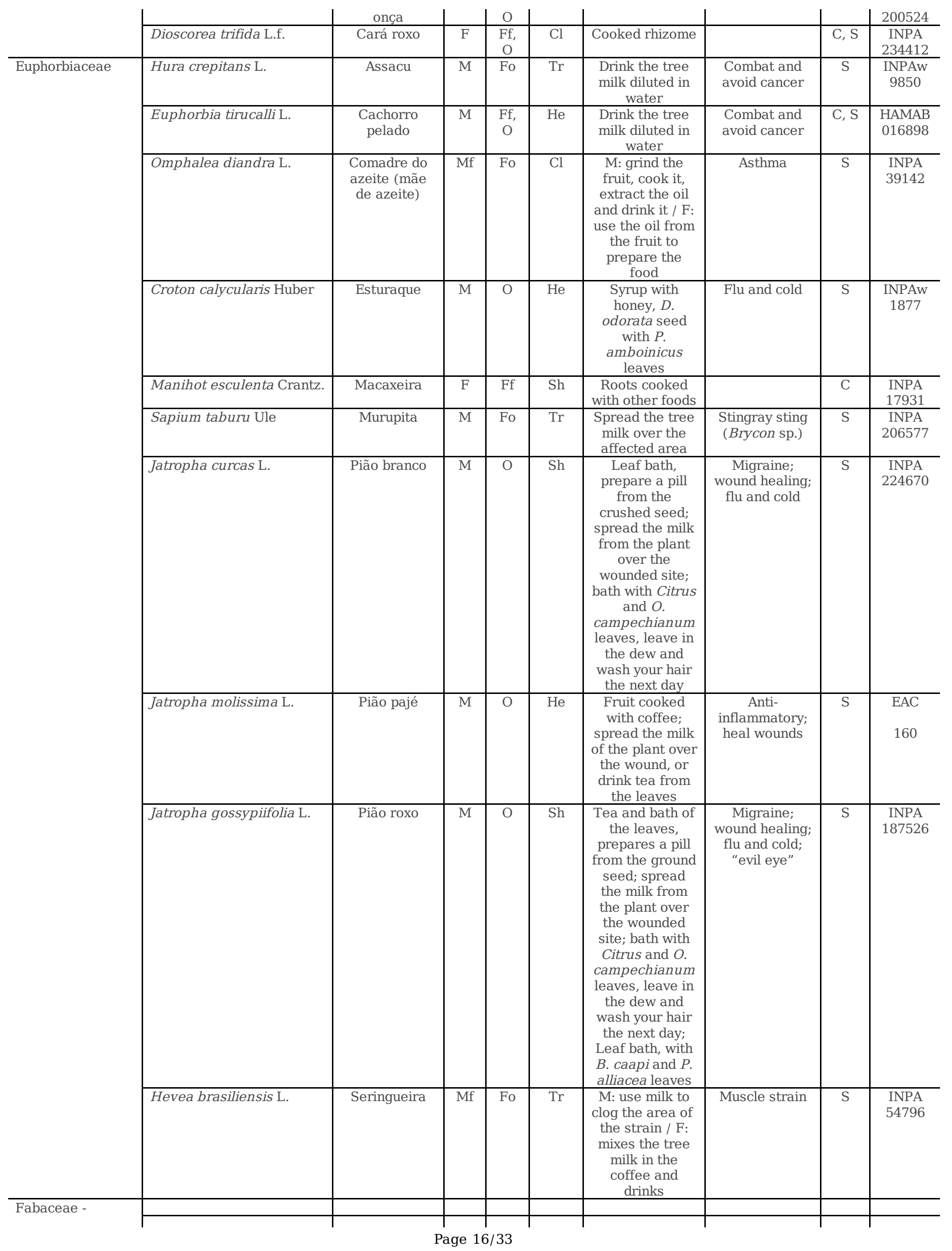




\begin{tabular}{|c|c|c|c|c|c|c|c|c|c|}
\hline \multirow[t]{7}{*}{ caesalpinioideae } & $\begin{array}{l}\text { Vouacapoua americana } \\
\text { Aubl. }\end{array}$ & Acapu & M & Fo & $\operatorname{Tr}$ & Tree bark tea & Amoeba & S & $\begin{array}{c}\text { INPA } \\
266591\end{array}$ \\
\hline & Ormosia coutinhoi Ducke & Buiuçú & Mf & Fo & $\mathrm{Sh}$ & $\begin{array}{c}\text { M: put tree bark } \\
\text { it in the water } \\
\text { until it gets } \\
\text { colored and } \\
\text { drink it / F: } \\
\text { fresh fruit }\end{array}$ & "evil eye" & $\mathrm{S}$ & $\begin{array}{c}\text { INPA } \\
140000\end{array}$ \\
\hline & $\begin{array}{l}\text { Copaifera } \\
\text { langsdorfii Desf. }\end{array}$ & Copaíba & $\mathrm{M}$ & Fo & $\operatorname{Tr}$ & $\begin{array}{l}\text { Beverage of the } \\
\text { tree oil with } E \text {. } \\
\text { guineensis } \\
\text { roots and honey }\end{array}$ & Gastritis & S & $\begin{array}{l}\text { INPA } \\
74512\end{array}$ \\
\hline & Hymenaea courbaril L. & Jatobá (jutaí) & Mf & Fo & $\operatorname{Tr}$ & $\begin{array}{c}\text { M: beverage } \\
\text { with } D \text {. odorata } \\
\text { and } B \text {. orellana } \\
\text { seeds, } Z \text {. mioga } \\
\text { and honey / F: } \\
\text { fresh fruit }\end{array}$ & $\begin{array}{l}\text { Flu, cough and } \\
\text { pneumonia }\end{array}$ & S & $\begin{array}{c}\text { INPA } \\
143023\end{array}$ \\
\hline & $\begin{array}{l}\text { Caesalpinia ferrea var. } \\
\text { cearensis Huber. }\end{array}$ & Jucá & $\mathrm{M}$ & Fo & $\operatorname{Tr}$ & Leaf tea & Stomachache & S & $\begin{array}{c}\text { IAN } \\
11254\end{array}$ \\
\hline & Senna alata (L.) Roxb. & Mata-pasto & $\mathrm{M}$ & $\mathrm{Rf}$ & $\mathrm{Sh}$ & Flower tea & Worm & S & $\begin{array}{c}\text { INPA } \\
192128\end{array}$ \\
\hline & Tamarindus indica L. & Tamarindo & $\mathrm{F}$ & Fo & $\operatorname{Tr}$ & Fresh fruit & & S & $\begin{array}{l}\text { INPA } \\
40979 \\
\end{array}$ \\
\hline \multirow{10}{*}{$\begin{array}{l}\text { Fabaceae - } \\
\text { faboideae }\end{array}$} & & & & & & & & & \\
\hline & $\begin{array}{l}\text { Dalbergia subcymosa } \\
\text { Ducke. }\end{array}$ & Cipó verônica & $\mathrm{M}$ & $\begin{array}{c}\text { Rf, } \\
\mathrm{O}\end{array}$ & Sh & $\begin{array}{l}\text { Beverage: bark } \\
\text { with and leaves } \\
\text { of } P \text {. } \\
\text { americana, } F \text {. } \\
\text { chica and of } G \text {. } \\
\text { hirsutum; or } \\
\text { with bark of } \\
\text { espinheira } \\
\text { santa, } U \text {. } \\
\text { tomentosa and } \\
S . \text { adstringens }\end{array}$ & $\begin{array}{l}\text { Gastritis and } \\
\text { anemia }\end{array}$ & S & $\begin{array}{c}\text { INPA } \\
248892\end{array}$ \\
\hline & $\begin{array}{l}\text { Dipteryx odorata (Aubl.) } \\
\text { Wild. }\end{array}$ & Cumaru & $\mathrm{M}$ & Fo & $\operatorname{Tr}$ & $\begin{array}{l}\text { Beverage of } H \text {. } \\
\text { courbaril bark, } \\
B \text {. orellana } \\
\text { seeds, } Z \text {. mioga } \\
\text { and honey; } \\
\text { syrup with } \\
\text { honey, leaves } C \text {. } \\
\text { calycularis and } \\
P \text {. amboinicus }\end{array}$ & $\begin{array}{c}\text { Flu, cough, } \\
\text { pneumonia and } \\
\text { could }\end{array}$ & S & $\begin{array}{c}\text { INPA } \\
171369\end{array}$ \\
\hline & $\begin{array}{l}\text { Bauhinia rutilans Spruce } \\
\text { ex Benth. }\end{array}$ & $\begin{array}{c}\text { Escada de } \\
\text { jabuti }\end{array}$ & $\mathrm{M}$ & Fo & $\mathrm{Cl}$ & $\begin{array}{c}\text { Tea or beverage } \\
\text { of vine }\end{array}$ & $\begin{array}{l}\text { Aches over the } \\
\text { body }\end{array}$ & $\mathrm{S}$ & $\begin{array}{c}\text { HAMAB } \\
9721\end{array}$ \\
\hline & Vicia faba L. & Faveira & $\mathrm{M}$ & Fo & $\operatorname{Tr}$ & $\begin{array}{l}\text { Squeeze the } \\
\text { seed oil into the } \\
\text { skin with } \\
\text { ringworm } \\
\end{array}$ & Ringworm & S & $\begin{array}{l}\text { MAR } \\
1293\end{array}$ \\
\hline & Bauhinia splendens Kunth & Macaco cipó & M & Fo & $\mathrm{Cl}$ & $\begin{array}{c}\text { Use milk to clog } \\
\text { the area of the } \\
\text { strain }\end{array}$ & Muscle strain & $\mathrm{S}$ & $\begin{array}{l}\text { INPA } \\
88854 \\
\end{array}$ \\
\hline & Erythrina falcata Benth. & Molongó & Mf & Fo & $\operatorname{Tr}$ & $\begin{array}{l}\text { M: spread the } \\
\text { tree milk on the } \\
\text { affected area / } \\
\text { F: fresh fruit }\end{array}$ & $\begin{array}{l}\text { Eliminate the } \\
\text { skin bug }\end{array}$ & $\mathrm{S}$ & $\begin{array}{c}\mathrm{UB} \\
141887\end{array}$ \\
\hline & Pterocarpus rohrii Vahl & Mututi & $\mathrm{M}$ & Fo & $\operatorname{Tr}$ & $\begin{array}{l}\text { Tea; put it in the } \\
\text { water until it } \\
\text { gets colored } \\
\text { and drink it } \\
\end{array}$ & $\begin{array}{c}\text { Anti- } \\
\text { inflammatory }\end{array}$ & $\mathrm{S}$ & $\begin{array}{l}\text { INPA } \\
3358\end{array}$ \\
\hline & Canavalia boliviana Piper. & $\begin{array}{l}\text { Papo de } \\
\text { mutum }\end{array}$ & $\mathrm{F}$ & Fo & $\operatorname{Tr}$ & Fresh fruit & & S & $\begin{array}{c}\text { EAC } \\
24200 \\
\end{array}$ \\
\hline & $\begin{array}{l}\text { Ateleia glazioveana } \\
\text { Baillon }\end{array}$ & Timbó & $\mathrm{M}$ & $\mathrm{O}$ & $\mathrm{He}$ & $\begin{array}{c}\text { Rub the leaf } \\
\text { milk over the } \\
\text { distended area }\end{array}$ & Muscle strain & S & $\begin{array}{l}\text { INPA } \\
2626 \\
\end{array}$ \\
\hline \multirow[t]{2}{*}{$\begin{array}{l}\text { Fabaceae - } \\
\text { mimosoideae }\end{array}$} & $\begin{array}{l}\text { Pentaclethra } \\
\text { macroloba Wild. Kuntze }\end{array}$ & Pracaxi & $\mathrm{M}$ & Fo & $\operatorname{Tr}$ & $\begin{array}{l}\text { Apply the oil } \\
\text { over the } \\
\text { infection } \\
\end{array}$ & Skin infection & $\mathrm{S}$ & $\begin{array}{l}\text { INPA } \\
1356 \\
\end{array}$ \\
\hline & $\begin{array}{l}\text { Stryphnodendron } \\
\text { adstringens (Mart.) Covile }\end{array}$ & Barbatimão & M & Fo & $\operatorname{Tr}$ & $\begin{array}{l}\text { Beverage of the } \\
\text { bark tree with } \\
\text { bark of }\end{array}$ & Gastritis & S & $\begin{array}{l}\text { INPA } \\
220296\end{array}$ \\
\hline
\end{tabular}




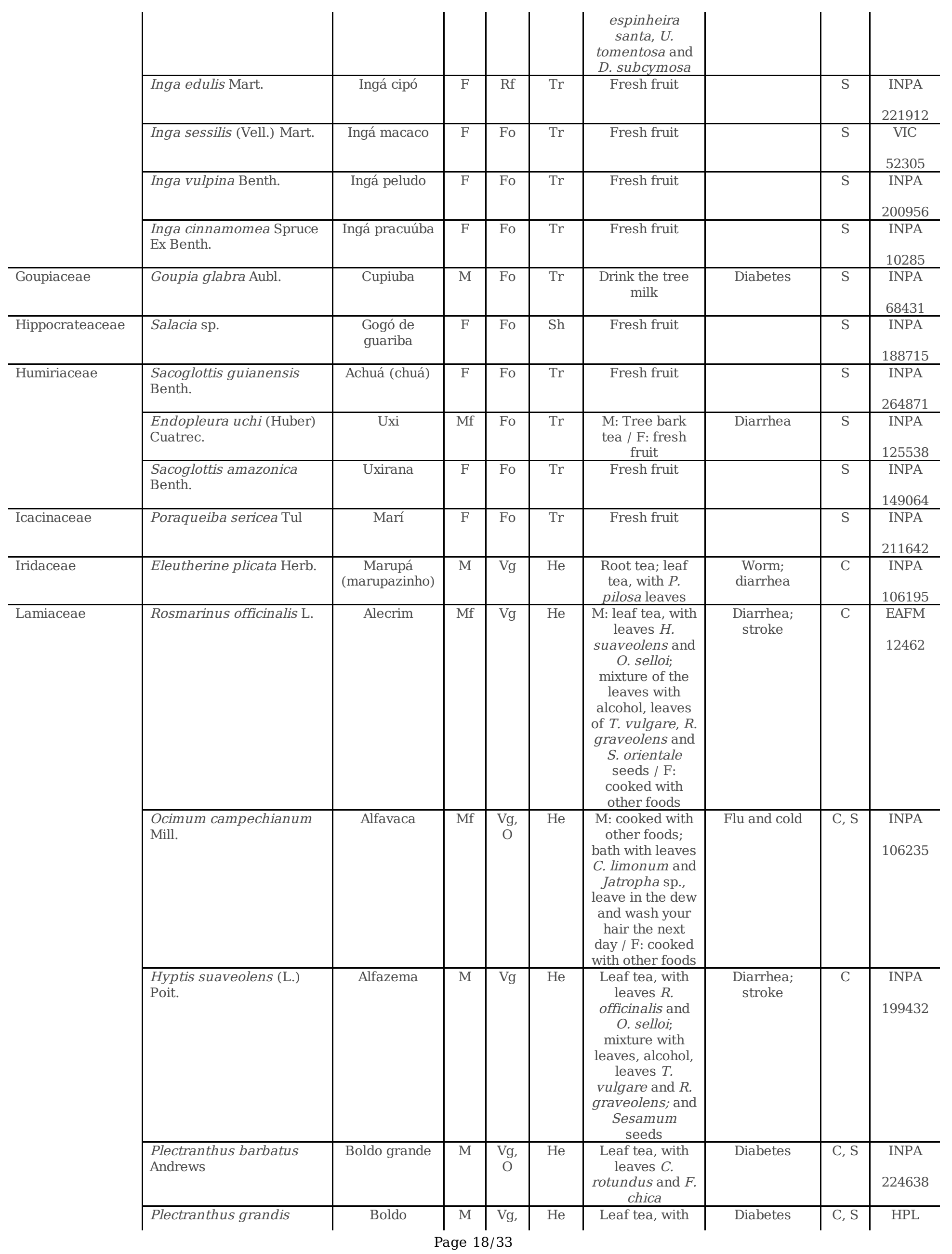




\begin{tabular}{|c|c|c|c|c|c|c|c|c|c|}
\hline & (Cramer) R. Willense & pequeno & & $\mathrm{O}$ & & $\begin{array}{c}\text { leaves } C . \\
\text { rotundus and } F \text {. } \\
\text { chica }\end{array}$ & & & 3629 \\
\hline & Marrubium vulgare L. & Desinflama & $\mathrm{M}$ & $\begin{array}{l}\mathrm{Vg} \\
\mathrm{O}\end{array}$ & $\mathrm{He}$ & Leaf tea & Tiredness & C, S & $\begin{array}{c}\text { IAN } \\
167767\end{array}$ \\
\hline & Ocimum selloi Benth. & $\begin{array}{c}\text { Elixir } \\
\text { paregórico }\end{array}$ & $\mathrm{M}$ & $\begin{array}{l}\mathrm{Vg} \\
\mathrm{O}\end{array}$ & $\mathrm{He}$ & $\begin{array}{l}\text { Leaf tea, with } \\
\text { leaves } R . \\
\text { officinalis and } \\
R \text {. officinalis; } \\
\text { mixture with } \\
\text { leaves, alcohol, } \\
\text { leaves } T \text {. } \\
\text { vulgare and } R \text {. } \\
\text { graveolens; and } \\
\text { Sesamum } \\
\text { seeds }\end{array}$ & $\begin{array}{l}\text { Diarrhea; } \\
\text { stroke }\end{array}$ & C, S & $\begin{array}{c}\text { IAC } \\
44399\end{array}$ \\
\hline & Melissa officinalis L. & Erva cidreira & Mf & $\begin{array}{c}\mathrm{Vg}, \\
\mathrm{O}\end{array}$ & $\mathrm{He}$ & $\begin{array}{c}\text { M: leaf tea / F: } \\
\text { cooked with } \\
\text { other foods }\end{array}$ & Tranquilizer & C, S & $\begin{array}{c}\text { IAN } \\
35950\end{array}$ \\
\hline & $\begin{array}{l}\text { Plectranthus amboinicus } \\
\text { (Lour.) Spreng. }\end{array}$ & $\begin{array}{c}\text { Hortelã } \\
\text { grande }\end{array}$ & Mf & $\begin{array}{l}\mathrm{Vg} \\
\mathrm{O}\end{array}$ & $\mathrm{He}$ & $\begin{array}{l}\text { M: syrup with } \\
\text { honey, } D \text {. } \\
\text { odorata seeds } \\
\text { and } C \text {. } \\
\text { calycularis } \\
\text { leaves / F: } \\
\text { cooked with } \\
\text { other foods }\end{array}$ & Flu and cold & C, S & $\begin{array}{c}\text { INPA } \\
268057\end{array}$ \\
\hline & Mentha $x$ villosa Huds. & Hortelanzinho & Mf & $\begin{array}{l}\mathrm{Vg} \\
\mathrm{O}\end{array}$ & $\mathrm{He}$ & $\begin{array}{l}\text { M: syrup with } \\
\text { honey, } D \text {. } \\
\text { odorata seeds } \\
\text { and } C \text {. } \\
\text { calycularis } \\
\text { leaves / F: } \\
\text { cooked with } \\
\text { other foods }\end{array}$ & Flu and cold & $\mathrm{C}, \mathrm{S}$ & $\begin{array}{c}\text { EAC } \\
54138\end{array}$ \\
\hline & Ocimum basilicum L. & Manjericão & Mf & $\begin{array}{l}\mathrm{Vg} \\
\mathrm{O}\end{array}$ & $\mathrm{He}$ & $\begin{array}{l}\text { M: leaf tea and } \\
\text { bath to wash } \\
\text { your head / F: } \\
\text { cooked with } \\
\text { other foods }\end{array}$ & Flu and cold & $\mathrm{C}, \mathrm{S}$ & $\begin{array}{l}\text { HFSL } \\
2735\end{array}$ \\
\hline & Origanum vulgare L. & Manjerona & $\mathrm{M}$ & $\begin{array}{c}\mathrm{Vg} \\
\mathrm{O}\end{array}$ & $\mathrm{He}$ & Leaf tea & Headache & C, S & $\begin{array}{c}\text { INPA } \\
147733\end{array}$ \\
\hline & Pogostemon cablin Benth. & Oriza & $\mathrm{M}$ & $\begin{array}{l}\mathrm{Vg} \\
\mathrm{O}\end{array}$ & $\mathrm{He}$ & Leaf tea & $\begin{array}{l}\text { Aches over the } \\
\text { body }\end{array}$ & $\mathrm{C}, \mathrm{S}$ & $\begin{array}{r}\text { INPA } \\
187521\end{array}$ \\
\hline & $\begin{array}{l}\text { Tetradenia riparia } \\
\text { (Hochst.) Codd }\end{array}$ & Pluma & $\mathrm{M}$ & $\begin{array}{l}\text { Vg, } \\
\mathrm{O}\end{array}$ & $\mathrm{He}$ & Leaf tea & Stomachache & C, S & $\begin{array}{l}\text { EAFM } \\
12123\end{array}$ \\
\hline & $\begin{array}{l}\text { Scutellaria agrestis A. St.- } \\
\text { Hil. ex Benth. }\end{array}$ & $\begin{array}{c}\text { Trevo roxo } \\
\text { (panana) }\end{array}$ & $\mathrm{M}$ & $\begin{array}{l}\mathrm{Vg} \\
\mathrm{O}\end{array}$ & $\mathrm{He}$ & $\begin{array}{c}\text { Squeeze the } \\
\text { juice from the } \\
\text { leaves on the } \\
\text { ear }\end{array}$ & Ear pain & $\mathrm{C}, \mathrm{S}$ & $\begin{array}{r}\text { INPA } \\
235447\end{array}$ \\
\hline & Mentha spicata L. & Vique grande & $\mathrm{M}$ & $\begin{array}{l}\mathrm{Vg} \\
\mathrm{O}\end{array}$ & $\mathrm{He}$ & Leaf tea & Headache & $\mathrm{C}, \mathrm{S}$ & $\begin{array}{c}\text { INPA } \\
233360\end{array}$ \\
\hline & Mentha arvensis L. & Vique pequeno & $\mathrm{M}$ & $\begin{array}{l}\mathrm{Vg} \\
\mathrm{O}\end{array}$ & $\mathrm{He}$ & Leaf tea & Headache & C, S & $\begin{array}{c}\text { IAN } \\
112431\end{array}$ \\
\hline Lauraceae & Persea americana Mill. & Abacate & Mf & $\mathrm{O}$ & $\operatorname{Tr}$ & $\begin{array}{c}\text { M: leaf tea; } \\
\text { beverage of } \\
\text { leaves with } D \text {. } \\
\text { subcymosa } \\
\text { bark, and } F \text {. } \\
\text { chica and } G \text {. } \\
\text { hirsutum leaves } \\
\text { / F: fresh fruit } \\
\text { and juice }\end{array}$ & $\begin{array}{c}\text { Rheumatism; } \\
\text { gastritis and } \\
\text { anemia }\end{array}$ & $\mathrm{C}$ & $\begin{array}{c}\text { INPA } \\
280645\end{array}$ \\
\hline & $\begin{array}{l}\text { Cinnamomum zeylanicum } \\
\text { Blume }\end{array}$ & Canela & Mf & $\mathrm{O}$ & $\operatorname{Tr}$ & Leaf tea $(\mathrm{M} / \mathrm{F})$ & Tranquilizer & $\mathrm{S}$ & $\begin{array}{l}\text { EAFM } \\
13320\end{array}$ \\
\hline & Aniba canelilla (Kunth) & Preciosa & Mf & $\mathrm{O}$ & $\operatorname{Tr}$ & Leaf tea $(\mathrm{M} / \mathrm{F})$ & Stomachache & $\mathrm{S}$ & $\begin{array}{c}\text { IAN } \\
146413\end{array}$ \\
\hline Lecythidaceae & Bertholletia excelsa & Castanha-do- & Mf & Fo & $\operatorname{Tr}$ & M: put tree bark & Amoeba; & S & INPAw \\
\hline
\end{tabular}




\begin{tabular}{|c|c|c|c|c|c|c|c|c|c|}
\hline & H.B.K. & brasil & & & & $\begin{array}{l}\text { it in the water } \\
\text { until it gets } \\
\text { colored and } \\
\text { drink it; leaf tea } \\
\text { with } C \text {. citratus } \\
\text { and } E \text {. oleracea } \\
\text { root, and } C \text {. } \\
\text { nucifera / F: } \\
\text { fresh almonds }\end{array}$ & hepatitis & & 7785 \\
\hline & $\begin{array}{l}\text { Couroupita guianensis } \\
\text { Aubl. }\end{array}$ & Curupita & $\mathrm{M}$ & Fo & $\operatorname{Tr}$ & $\begin{array}{l}\text { Spread the tree } \\
\text { milk under the } \\
\text { affected area }\end{array}$ & $\begin{array}{l}\text { Stingray sting } \\
\text { (Brycon sp.), } \\
\text { scorpion and } \\
\text { snake } \\
\end{array}$ & $\mathrm{S}$ & $\begin{array}{l}\text { INPA } \\
15960\end{array}$ \\
\hline & Lecythis pisonis Cambess. & Sapucaia & Mf & Fo & $\operatorname{Tr}$ & $\begin{array}{l}\text { M: put tree bark } \\
\text { it in the water } \\
\text { until it gets } \\
\text { colored and } \\
\text { under the } \\
\text { affected area - / } \\
\text { F: fresh } \\
\text { almonds } \\
\end{array}$ & Ringworm & S & $\begin{array}{c}\text { INPA } \\
161763\end{array}$ \\
\hline Loganiaceae & Spigelia anthelmia L. & Lombrigueira & $\mathrm{M}$ & Fo & $\mathrm{He}$ & Leaf tea & Worm & $\mathrm{S}$ & $\begin{array}{c}\text { INPA } \\
104116\end{array}$ \\
\hline \multirow[t]{2}{*}{ Malpighiaceae } & $\begin{array}{l}\text { Banisteria caapi (Spruce } \\
\text { ex Griseb.) }\end{array}$ & Cabi & $\mathrm{M}$ & Fo & $\operatorname{Tr}$ & $\begin{array}{c}\text { Leaf tea with } J \text {. } \\
\text { gossypiifolia } \\
\text { and } P . \text { alliacea } \\
\text { leaves }\end{array}$ & "evil eye" & S & $\begin{array}{l}\text { RFA } \\
5243\end{array}$ \\
\hline & $\begin{array}{l}\text { Byrsonima crassifolia (L.) } \\
\text { Kunth }\end{array}$ & Muruci & $\mathrm{F}$ & Fo & $\operatorname{Tr}$ & $\begin{array}{l}\text { Fresh fruit and } \\
\text { juice }\end{array}$ & & S & $\begin{array}{r}\text { INPA } \\
187250 \\
\end{array}$ \\
\hline \multirow[t]{7}{*}{ Malvaceae } & Gossypium hirsutum L. & $\begin{array}{l}\text { Algodão } \\
\text { branco }\end{array}$ & $\mathrm{M}$ & $\mathrm{O}$ & Sh & $\begin{array}{l}\text { Beverage of } \\
\text { leaf with bark } \\
D . \text { subcymosa, } \\
\text { F. chica and } P \text {. } \\
\text { americana; } \\
\text { grind the seed } \\
\text { with milk and } \\
\text { drink it }\end{array}$ & $\begin{array}{c}\text { Gastritis, } \\
\text { anemia; babies } \\
\text { vomiting }\end{array}$ & S & $\begin{array}{c}\text { INPA } \\
106310\end{array}$ \\
\hline & $\begin{array}{l}\text { Herrania mariae (Mart.) } \\
\text { Decne. ex Goudot }\end{array}$ & $\begin{array}{l}\text { Cacauí (cacau } \\
\text { jacaré) }\end{array}$ & $\mathrm{F}$ & Fo & $\operatorname{Tr}$ & Fresh fruit & & $\mathrm{S}$ & $\begin{array}{l}\text { INPA } \\
3788\end{array}$ \\
\hline & $\begin{array}{l}\text { Theobroma subincanum } \\
\text { Mart. }\end{array}$ & Cupuí & $\mathrm{F}$ & Fo & $\operatorname{Tr}$ & Fresh fruit & & S & $\begin{array}{c}\text { INPA } \\
200343 \\
\end{array}$ \\
\hline & Althaea officinalis L. & Malvarisco & $\mathrm{M}$ & $\mathrm{Vg}$ & $\mathrm{He}$ & $\begin{array}{l}\text { Grind the leaf, } \\
\text { spread the gel } \\
\text { on the place and } \\
\text { tie with cloth }\end{array}$ & $\begin{array}{l}\text { Stop injury } \\
\text { blood }\end{array}$ & $\mathrm{C}$ & $\begin{array}{c}\text { IAC } \\
56164\end{array}$ \\
\hline & Pachira aquatica Aubl. & Mamorana & $\mathrm{F}$ & Fo & $\operatorname{Tr}$ & Boiled almonds & & S & $\begin{array}{c}\text { INPA } \\
212659 \\
\end{array}$ \\
\hline & $\begin{array}{l}\text { Abelmoschus esculentus } \\
\text { L. Moench }\end{array}$ & Quiabo & Mf & $\begin{array}{l}\text { Ff, } \\
\mathrm{Vg}\end{array}$ & Sh & $\begin{array}{c}\text { M: dried fruit } \\
\text { tea / F: cooked } \\
\text { with other foods }\end{array}$ & $\begin{array}{l}\text { Postpartum } \\
\text { treatment }\end{array}$ & $\mathrm{C}$ & $\begin{array}{l}\text { EAFM } \\
10867 \\
\end{array}$ \\
\hline & Hibiscus sabdariffa L. & Vinagreira & Mf & $\mathrm{O}$ & Sh & $\begin{array}{c}\text { M: dried fruit } \\
\text { tea / F: cooked } \\
\text { with other foods }\end{array}$ & Tranquilizer & S & $\begin{array}{l}\text { INPA } \\
57076 \\
\end{array}$ \\
\hline Marantaceae & $\begin{array}{l}\text { Calathea allouia (Aubl.) } \\
\text { Lindl }\end{array}$ & Ariá & $\mathrm{F}$ & Ff & $\mathrm{He}$ & Cooked rhizome & & $\mathrm{C}$ & $\begin{array}{l}\text { EAFM } \\
5247 \\
\end{array}$ \\
\hline Melastomataceae & Mouriri grandiflora D.C. & Camutim & $\mathrm{F}$ & Fo & Sh & Fresh fruit & & S & $\begin{array}{r}\text { INPA } \\
146192 \\
\end{array}$ \\
\hline \multirow[t]{2}{*}{ Meliaceae } & Carapa guianensis Aubl. & Andiroba & $\mathrm{M}$ & Fo & $\operatorname{Tr}$ & $\begin{array}{c}\text { Beverage of oil } \\
\text { extracted from } \\
\text { the stem with } \\
\text { bee honey and } \\
\text { leaves of } A \text {. } \\
\text { oleracea }\end{array}$ & $\begin{array}{l}\text { Sore throat } \\
\text { and flu }\end{array}$ & S & $\begin{array}{l}\text { INPA } \\
158520\end{array}$ \\
\hline & $\begin{array}{l}\text { Guarea guidonia (L.) } \\
\text { Sleumer }\end{array}$ & Jataúba & $\mathrm{M}$ & Fo & $\operatorname{Tr}$ & $\begin{array}{c}\text { Cooked root } \\
\text { with other foods }\end{array}$ & $\begin{array}{l}\text { To clean the } \\
\text { intestine }\end{array}$ & $\mathrm{S}$ & $\begin{array}{r}\text { INPA } \\
118374 \\
\end{array}$ \\
\hline Menispermaceae & Abuta grandifolia L. & Bôta & $\mathrm{M}$ & $\mathrm{Rf}$ & $\mathrm{Cl}$ & $\begin{array}{c}\text { Leaf tea with } P \text {. } \\
\text { angulata root, } \\
Q . \text { amara leaf }\end{array}$ & Malaria & $\mathrm{S}$ & $\begin{array}{l}\text { INPA } \\
94362\end{array}$ \\
\hline
\end{tabular}




\begin{tabular}{|c|c|c|c|c|c|c|c|c|c|}
\hline & & & & & & $\begin{array}{l}\text { and } L \text {. } \\
\text { operculata fruit } \\
\text { dried }\end{array}$ & & & \\
\hline Monimiaceae & Siparuna guianensis L. & Capitiú & $\mathrm{M}$ & $\mathrm{Vg}$ & $\mathrm{He}$ & $\begin{array}{l}\text { Tree bark tea; } \\
\text { bath with bark } \\
\text { of nazarana } \\
\text { tree and } C \text {. } \\
\text { deodara with } C \text {. } \\
\text { cujete leaf }\end{array}$ & $\begin{array}{c}\text { Flu and } \\
\text { headache; } \\
\text { fever and “evil } \\
\text { eye” }\end{array}$ & $\mathrm{C}$ & $\begin{array}{c}\text { INPA } \\
165827\end{array}$ \\
\hline \multirow[t]{4}{*}{ Moraceae } & Brosimum potabile Ducke. & Amapá doce & Mf & Fo & $\operatorname{Tr}$ & $\begin{array}{l}\text { Collect the milk } \\
\text { from the tree, } \\
\text { beat it to } \\
\text { remove the } \\
\text { foam and drink } \\
\text { it, mashed or } \\
\text { with coffee } \\
(\mathrm{M} / \mathrm{F}) \\
\end{array}$ & Gastritis & $\mathrm{S}$ & $\begin{array}{l}\text { INPA } \\
7638\end{array}$ \\
\hline & Ficus insipida Willd. & $\begin{array}{c}\text { Apuí } \\
\text { (caxinguba) }\end{array}$ & $\mathrm{M}$ & Fo & $\operatorname{Tr}$ & $\begin{array}{c}\text { Use milk to clog } \\
\text { the area of the } \\
\text { strain }\end{array}$ & Muscle strain & $\mathrm{S}$ & $\begin{array}{l}\text { INPA } \\
39967 \\
\end{array}$ \\
\hline & $\begin{array}{l}\text { Artocarpus camansi } \\
\text { Blanco }\end{array}$ & Fruta pão & Mf & Fo & $\operatorname{Tr}$ & $\begin{array}{l}\text { M: Use milk to } \\
\text { clog the area of } \\
\text { the strain / F: } \\
\text { cooked fruit }\end{array}$ & Muscle strain & $\mathrm{S}$ & $\begin{array}{c}\text { INPA } \\
280857\end{array}$ \\
\hline & $\begin{array}{l}\text { Artocarpus heterophyllus } \\
\text { Lam. }\end{array}$ & Jaca & $\mathrm{F}$ & $\mathrm{O}$ & $\operatorname{Tr}$ & Fresh fruit & & $\mathrm{S}$ & $\begin{array}{c}\text { INPA } \\
192145\end{array}$ \\
\hline \multirow[t]{2}{*}{ Musaceae } & Musa paradisiaca L. & $\begin{array}{c}\text { Banana } \\
\text { (bananeira) }\end{array}$ & Mf & $\mathrm{O}$ & $\mathrm{Sh}$ & $\begin{array}{c}\text { M: Bath: dry } \\
\text { leaves with } E \text {. } \\
\text { oleracea and } C \text {. } \\
\text { nucifera, with } \\
M \text {. indica peel } \\
\text { and } C \text {. citratus } \\
\text { leaf / F: fresh } \\
\text { fruit }\end{array}$ & $\begin{array}{l}\text { Bathe woman } \\
\text { after childbirth }\end{array}$ & $\mathrm{C}$ & $\begin{array}{c}\text { INPA } \\
199519\end{array}$ \\
\hline & Musa acuminata L. & Banana roxa & Mf & $\mathrm{O}$ & Sh & $\begin{array}{l}\text { M: Bath: dry } \\
\text { leaves with } E \text {. } \\
\text { oleracea and } C \text {. } \\
\text { nucifera, with } \\
M \text {. indica peel } \\
\text { and } C \text {. citratus } \\
\text { leaf; beverage } \\
\text { with mangangá } \\
\text { and } C \text {. spicatus } \\
\text { leaves and } V . \\
\text { surinamensis } \\
\text { bark / F: fresh } \\
\text { fruit }\end{array}$ & $\begin{array}{c}\text { Bathe woman } \\
\text { after childbirth; } \\
\text { uterus } \\
\text { infection }\end{array}$ & $\mathrm{C}$ & $\begin{array}{l}\text { OUPR } \\
30362\end{array}$ \\
\hline Myristicaceae & $\begin{array}{l}\text { Virola surinamensis (Rol. } \\
\text { ex Rottb.) Warb. }\end{array}$ & $\begin{array}{c}\text { Virola, } \\
\text { ucuuba, } \\
\text { bucuuba }\end{array}$ & $\mathrm{M}$ & Fo & $\operatorname{Tr}$ & $\begin{array}{c}\text { Beverage of the } \\
\text { bark tree with } \\
C . \text { spicatus } \\
\text { leaves and } M \text {. } \\
\text { acuminata } \\
\text { mangangá }\end{array}$ & $\begin{array}{l}\text { Uterus } \\
\text { infection }\end{array}$ & $\mathrm{S}$ & $\begin{array}{l}\text { INPA } \\
57328\end{array}$ \\
\hline \multirow[t]{6}{*}{ Myrtaceae } & $\begin{array}{l}\text { Syzygium cumini (L.) } \\
\text { Skeels }\end{array}$ & Ameixa & Mf & $\mathrm{O}$ & $\operatorname{Tr}$ & $\begin{array}{l}\text { M: tree bark tea } \\
\text { / F: fresh fruit }\end{array}$ & Diarrhea & $\mathrm{S}$ & $\begin{array}{c}\text { INPA } \\
268285\end{array}$ \\
\hline & $\begin{array}{l}\text { Psidium cattleianum } \\
\text { Sabine }\end{array}$ & Araçá & $\mathrm{F}$ & $\mathrm{O}$ & Sh & Fresh fruit & & $\mathrm{S}$ & $\begin{array}{l}\text { EAFM } \\
12223 \\
\end{array}$ \\
\hline & $\begin{array}{l}\text { Syzygium aromaticum } \\
\text { (L.) Merr. \& L.M. Perry }\end{array}$ & Cravo arvore & $\mathrm{Mf}$ & Fo & $\operatorname{Tr}$ & $\begin{array}{l}\text { Tree bark tea } \\
(\mathrm{M} / \mathrm{F})\end{array}$ & $\begin{array}{l}\text { Stomachache, } \\
\text { intestinal } \\
\text { constipation } \\
\end{array}$ & $\mathrm{S}$ & $\begin{array}{l}\text { IAC } \\
5345 \\
\end{array}$ \\
\hline & $\begin{array}{l}\text { Eugenia victoriana } \\
\text { Cuatrec. }\end{array}$ & Ginja & $\mathrm{F}$ & $\mathrm{Rf}$ & $\operatorname{Tr}$ & Fresh fruit & & $\mathrm{S}$ & $\begin{array}{l}\text { HPL } \\
6127\end{array}$ \\
\hline & Psidium guajava L. & Goiaba & Mf & $\mathrm{O}$ & $\mathrm{Sh}$ & $\begin{array}{l}\text { M: tree bark tea } \\
\text { / F: fresh fruit }\end{array}$ & Diarrhea & $\mathrm{S}$ & $\begin{array}{c}\text { INPA } \\
237204 \\
\end{array}$ \\
\hline & $\begin{array}{l}\text { Syzygium malaccense } \\
\text { (L.) Merr. \& L.M. Perry }\end{array}$ & Jambo & $\mathrm{F}$ & $\mathrm{O}$ & $\mathrm{Tr}$ & Fresh fruit & & $\mathrm{S}$ & $\begin{array}{c}\text { INPA } \\
214039\end{array}$ \\
\hline Olacaceae & $\begin{array}{l}\text { Ptychopetalum } \\
\text { uncinatum Anselmino }\end{array}$ & Marapuama & $\mathrm{M}$ & $\mathrm{Rf}$ & $\mathrm{Tr}$ & $\begin{array}{l}\text { Mixture of the } \\
\text { tree bark with } \\
\text { alcohol and } \\
\text { massage } \\
\end{array}$ & $\begin{array}{l}\text { Cramp and } \\
\text { rheumatism }\end{array}$ & $\mathrm{S}$ & $\begin{array}{l}\text { INPA } \\
95874\end{array}$ \\
\hline
\end{tabular}

Page 21/33 


\begin{tabular}{|c|c|c|c|c|c|c|c|c|c|}
\hline \multirow[t]{2}{*}{ Oxalidaceae } & Averrhoa carambola L. & Carambola & $\mathrm{F}$ & $\mathrm{O}$ & Sh & $\begin{array}{l}\text { Fresh fruit and } \\
\text { juice }\end{array}$ & & $\mathrm{S}$ & $\begin{array}{c}\text { INPA } \\
224146 \\
\end{array}$ \\
\hline & Averrhoa bilimbi L. & Limão caiana & $\mathrm{F}$ & $\mathrm{O}$ & Sh & Food flavoring & & $\mathrm{S}$ & $\begin{array}{c}\text { INPA } \\
146883\end{array}$ \\
\hline \multirow[t]{3}{*}{ Passifloraceae } & $\begin{array}{l}\text { Passiflora micropetala } \\
\text { Mart. ex Mast. }\end{array}$ & $\begin{array}{l}\text { Maracujá de } \\
\text { paca }\end{array}$ & F & $\mathrm{O}$ & $\mathrm{Cl}$ & Fresh fruit & & $\mathrm{S}$ & $\begin{array}{c}\text { EAFM } \\
279\end{array}$ \\
\hline & Passiflora nitida Kunth & $\begin{array}{l}\text { Maracujá do } \\
\text { mato (de } \\
\text { cheiro) }\end{array}$ & $\mathrm{F}$ & Fo & $\mathrm{Cl}$ & Fresh fruit & & $\mathrm{S}$ & $\begin{array}{c}\text { INPA } \\
154814 \\
\end{array}$ \\
\hline & $\begin{array}{l}\text { Passiflora quadrangularis } \\
\text { L. }\end{array}$ & $\begin{array}{c}\text { Maracujá } \\
\text { peroba }\end{array}$ & F & Ff & $\mathrm{Cl}$ & Fresh fruit & & $\mathrm{C}$ & $\begin{array}{l}\text { INPA } \\
69856 \\
\end{array}$ \\
\hline \multirow[t]{2}{*}{ Pedaliaceae } & Sesamum orientale L. & $\begin{array}{l}\text { Gergelim } \\
\text { branco }\end{array}$ & Mf & $\mathrm{Ff}$ & $\mathrm{He}$ & $\begin{array}{l}\text { M: mixture with } \\
\text { seeds, alcohol, } \\
\text { leaves from T. } \\
\text { vulgare, } R \text {. } \\
\text { graveolens and } \\
\text { R. officinalis / } \\
\text { F: make paçoca } \\
\text { from the seeds }\end{array}$ & $\begin{array}{l}\text { Diarrhea; } \\
\text { stroke }\end{array}$ & $\mathrm{C}$ & $\begin{array}{c}\text { EAFM } \\
4722\end{array}$ \\
\hline & Sesamum indicum L. & $\begin{array}{l}\text { Gergelim } \\
\text { preto }\end{array}$ & Mf & Ff & $\mathrm{He}$ & $\begin{array}{l}\text { M: mixture with } \\
\text { seeds, alcohol, } \\
\text { leaves from T. } \\
\text { vulgare, } R \text {. } \\
\text { graveolens and } \\
R . \text { officinalis / } \\
\text { F: make paçoca } \\
\text { from the seeds }\end{array}$ & $\begin{array}{l}\text { Diarrhea; } \\
\text { stroke }\end{array}$ & $\mathrm{C}$ & $\begin{array}{c}\text { INPA } \\
206973\end{array}$ \\
\hline \multirow[t]{2}{*}{ Phyllanthaceae } & Phyllanthus niruri L. & Quebra pedra & $\mathrm{M}$ & $\mathrm{Vg}$ & $\mathrm{He}$ & $\begin{array}{l}\text { Leaf tea (or } G \text {. } \\
\text { arborescens } \\
\text { leaves), leaves } \\
\text { from } A \text {. tenella, } \\
\text { C. spicatus and } \\
\text { A. muricata }\end{array}$ & $\begin{array}{l}\text { Urinary tract } \\
\text { infection, } \\
\text { kidney stones }\end{array}$ & $\mathrm{C}$ & $\begin{array}{c}\text { INPA } \\
193468\end{array}$ \\
\hline & Petiveria alliacea L. & Mucuracaá & $\mathrm{M}$ & $\mathrm{Vg}$ & $\mathrm{He}$ & $\begin{array}{c}\text { Leaf tea with } J \text {. } \\
\text { gossypiifolia } \\
\text { and B. caapi } \\
\text { leaves; } \\
\text { beverage with } \\
\text { leaves } \\
\end{array}$ & $\begin{array}{l}\text { "evil eye"; } \\
\text { gastritis }\end{array}$ & $\mathrm{C}$ & $\begin{array}{c}\text { INPA } \\
259176\end{array}$ \\
\hline Pinaceae & Cedrus deodara L. & Cedro & $\mathrm{M}$ & Fo & $\operatorname{Tr}$ & $\begin{array}{l}\text { Bark tea; bath: } \\
\text { tree bark, with } \\
\text { nazarana bark, } \\
\text { and leaves from } \\
\text { S. guianensis } \\
\text { and } C \text {. cujete }\end{array}$ & $\begin{array}{c}\text { Tranquilizer } \\
\text { and } \\
\text { Stomachache; } \\
\text { fever and "evil } \\
\text { eye” }\end{array}$ & $\mathrm{S}$ & $\begin{array}{c}\mathrm{ICN} \\
128901\end{array}$ \\
\hline \multirow[t]{2}{*}{ Piperaceae } & $\begin{array}{l}\text { Peperomia pellucida (L.) } \\
\text { Kunth }\end{array}$ & $\begin{array}{c}\text { Comida de } \\
\text { jabuti }\end{array}$ & Mf & $\begin{array}{c}\mathrm{Vg}, \\
\mathrm{O}\end{array}$ & $\mathrm{He}$ & $\begin{array}{c}\text { M: leaf and } \\
\text { stem tea / F: } \\
\text { sautéed with } \\
\text { other foods }\end{array}$ & Infection & $\mathrm{S}$ & $\begin{array}{l}\text { INPA } \\
33834\end{array}$ \\
\hline & $\begin{array}{l}\text { Piper callosum Ruiz \& } \\
\text { Pav. }\end{array}$ & Óleo elétrico & $\mathrm{M}$ & $\mathrm{O}$ & $\mathrm{He}$ & Leaf tea & $\begin{array}{l}\text { Migraine and } \\
\text { sting of insects }\end{array}$ & $\mathrm{S}$ & $\begin{array}{r}\text { INPA } \\
243162 \\
\end{array}$ \\
\hline Plantaginaceae & Scoparia dulcis L. & Vassourinha & $\mathrm{M}$ & $\mathrm{O}$ & $\mathrm{He}$ & $\begin{array}{l}\text { Squeeze the } \\
\text { juice from the } \\
\text { leaves on the } \\
\text { affected area }\end{array}$ & $\begin{array}{l}\text { Mycosis and } \\
\text { skin irritation }\end{array}$ & $\mathrm{S}$ & $\begin{array}{l}\text { INPA } \\
58086\end{array}$ \\
\hline \multirow[t]{3}{*}{ Poaceae } & Bambusa vulgaris Schrad. & Bambu & $\mathrm{M}$ & Fo & $\operatorname{Tr}$ & Leaf tea & $\begin{array}{c}\text { Arterial } \\
\text { hypertension }\end{array}$ & $\mathrm{S}$ & $\begin{array}{c}\text { IAN } \\
197468 \\
\end{array}$ \\
\hline & Saccharum spp. L. & Cana & Mf & Ff & $\mathrm{Sh}$ & $\begin{array}{c}\text { Drink the juice } \\
\text { from the stem } \\
(\mathrm{M} / \mathrm{F})\end{array}$ & $\begin{array}{l}\text { Malaise and } \\
\text { indisposition }\end{array}$ & $\mathrm{C}$ & $\begin{array}{c}\text { IAN } \\
62600 \\
\end{array}$ \\
\hline & $\begin{array}{l}\text { Cymbopogon citratus } \\
\text { (DC.) Stapf }\end{array}$ & $\begin{array}{c}\text { Capim } \\
\text { marinho } \\
\text { (capim santo) }\end{array}$ & Mf & $\mathrm{Vg}$ & $\mathrm{He}$ & $\begin{array}{c}\text { M: leaf bath } \\
\text { with dry leaves } \\
\text { from } M . \\
\text { paradisíaca, } E \text {. } \\
\text { oleracea and } C \text {. } \\
\text { nucifera, with } \\
M \text {. indica peel; } \\
\text { leaf tea from } C \text {. } \\
\text { winterianus } \\
\text { with } E \text {. oleracea }\end{array}$ & $\begin{array}{c}\text { Bathe woman } \\
\text { after childbirth; } \\
\text { hepatitis }\end{array}$ & $\mathrm{C}$ & $\begin{array}{c}\text { INPA } \\
268065\end{array}$ \\
\hline
\end{tabular}




\begin{tabular}{|c|c|c|c|c|c|c|c|c|c|}
\hline & & & & & & $\begin{array}{c}\text { root, and } C \text {. } \\
\text { nucifera and } B \text {. } \\
\text { excelsa peel / } \\
\text { F: leaf tea }\end{array}$ & & & \\
\hline & $\begin{array}{l}\text { Cymbopogon winterianus } \\
\text { Jowitt ex Bor }\end{array}$ & Eucalipto & $\mathrm{M}$ & $\mathrm{Vg}$ & $\mathrm{He}$ & $\begin{array}{l}\text { Leaf tea with } \\
\text { raiz } C \text {. citratus } \\
\text { and } E \text {. oleracea } \\
\text { root, and } C \text {. } \\
\text { nucifera and } B \text {. } \\
\text { excelsa peel }\end{array}$ & Hepatitis & $\mathrm{C}$ & $\begin{array}{c}\text { IAN } \\
194324\end{array}$ \\
\hline & Guadua weberbaueri Pilg. & Tabuqui & $\mathrm{M}$ & Fo & Sh & $\begin{array}{l}\text { Chew and } \\
\text { swallow the } \\
\text { apical bud of } \\
\text { the plant }\end{array}$ & Sting of insects & S & $\begin{array}{l}\text { INPA } \\
26135\end{array}$ \\
\hline \multirow[t]{2}{*}{ Portulacaceae } & Portulaca pilosa L. & Amor crescido & $\mathrm{M}$ & $\mathrm{Vg}$ & $\mathrm{He}$ & $\begin{array}{l}\text { leaf tea with } E \text {. } \\
\text { plicata leaves }\end{array}$ & Diarrhea & $\mathrm{C}$ & $\begin{array}{c}\text { INPA } \\
177381 \\
\end{array}$ \\
\hline & Portulaca grandiflora L. & Onze-horas & $\mathrm{M}$ & $\mathrm{Vg}$ & $\mathrm{He}$ & Leaf tea & $\begin{array}{c}\text { Arterial } \\
\text { hypertension }\end{array}$ & $\mathrm{C}$ & $\begin{array}{l}\text { INPA } \\
56716 \\
\end{array}$ \\
\hline Rhamnaceae & $\begin{array}{l}\text { Houvenia dulcis } \\
\text { Thunberg. }\end{array}$ & Pau doce & $\mathrm{M}$ & Fo & $\operatorname{Tr}$ & Bark tea & Headache & S & $\begin{array}{l}\text { MACK } \\
2691 \\
\end{array}$ \\
\hline Rosaceae & $\begin{array}{l}\text { Licania macrophylla } \\
\text { Benth. }\end{array}$ & Anauerá & $\mathrm{M}$ & Fo & $\operatorname{Tr}$ & $\begin{array}{l}\text { Put tree bark it } \\
\text { in the water } \\
\text { until it gets } \\
\text { colored and } \\
\text { drink it }\end{array}$ & $\begin{array}{l}\text { Stomachache } \\
\text { and amoeba }\end{array}$ & S & $\begin{array}{c}\text { IAN } \\
11348\end{array}$ \\
\hline \multirow[t]{6}{*}{ Rubiaceae } & Genipa americana L. & Jenipapo & Mf & Fo & $\operatorname{Tr}$ & $\begin{array}{l}\text { Fresh fruit and } \\
\text { juice }(\mathrm{M} / \mathrm{F})\end{array}$ & Cholesterol & S & $\begin{array}{l}\text { INPA } \\
1871 \\
\end{array}$ \\
\hline & Morinda citrifolia L. & Noni & $\mathrm{M}$ & $\mathrm{O}$ & Sh & Leaf tea & $\begin{array}{l}\text { Aches over the } \\
\text { body }\end{array}$ & S & $\begin{array}{c}\text { INPA } \\
237845 \\
\end{array}$ \\
\hline & $\begin{array}{l}\text { Calycophyllum } \\
\text { spruceanum (Benth.) K. }\end{array}$ & Pau mulato & $\mathrm{M}$ & $\mathrm{O}$ & $\operatorname{Tr}$ & Bark tea & $\begin{array}{l}\text { Stomachache } \\
\text { and amoeba }\end{array}$ & S & $\begin{array}{c}\text { INPA } \\
253827 \\
\end{array}$ \\
\hline & Alibertia sorbilis Ducke & Puruí & $\mathrm{F}$ & $\mathrm{Rf}$ & $\operatorname{Tr}$ & Fresh fruit & & $\mathrm{S}$ & $\begin{array}{l}\text { INPA } \\
16548\end{array}$ \\
\hline & Cinchona calisaya Weed. & Quinarana & $\mathrm{M}$ & Fo & $\operatorname{Tr}$ & Root tea & Fever & S & $\begin{array}{l}\text { IAC } \\
6280\end{array}$ \\
\hline & $\begin{array}{l}\text { Uncaria tomentosa (Willd) } \\
\text { D. C. }\end{array}$ & $\begin{array}{l}\text { Unha de gato } \\
\text { (jupindá) }\end{array}$ & $\mathrm{M}$ & $\mathrm{Rf}$ & $\mathrm{Cl}$ & $\begin{array}{l}\text { Beverage of the } \\
\text { bark of the vine } \\
\text { with bark from } \\
\text { espinheira } \\
\text { santa, } D . \\
\text { subcymosa and } \\
\text { S. adstringens }\end{array}$ & Gastritis & $\mathrm{S}$ & $\begin{array}{l}\text { INPA } \\
55269\end{array}$ \\
\hline \multirow[t]{6}{*}{ Rutaceae } & Ruta graveolens L. & Arruda & $\mathrm{M}$ & $\mathrm{Vg}$ & $\mathrm{He}$ & $\begin{array}{l}\text { Leaves mixture } \\
\text { with alcohol, } \\
\text { leaves from } T \text {. } \\
\text { vulgare and } O \text {. } \\
\text { campechianum, } \\
\text { and Sesamum } \\
\text { seeds }\end{array}$ & $\begin{array}{l}\text { Diarrhea; } \\
\text { stroke }\end{array}$ & $\mathrm{C}$ & $\begin{array}{l}\text { INPA } \\
100963\end{array}$ \\
\hline & Citrus sinensis L. Osb. & Laranja & Mf & $\mathrm{O}$ & $\operatorname{Tr}$ & $\begin{array}{l}\text { M: leaf tea with } \\
\text { A. esperanzae / } \\
\text { F: fresh fruit } \\
\text { and juice }\end{array}$ & Gases & S & $\begin{array}{c}\text { INPA } \\
161639\end{array}$ \\
\hline & Citrus aurantium L. & $\begin{array}{l}\text { Laranja da } \\
\text { terra }\end{array}$ & $\mathrm{M}$ & $\mathrm{O}$ & $\operatorname{Tr}$ & $\begin{array}{c}\text { Eat the fresh } \\
\text { fruit with bee } \\
\text { honey }\end{array}$ & Anemia & S & $\begin{array}{l}\text { INPA } \\
40936 \\
\end{array}$ \\
\hline & Citrus limettioides Tan & Lima & Mf & $\mathrm{O}$ & $\operatorname{Tr}$ & $\begin{array}{l}\text { M: tree bark tea } \\
\text { / F: fresh fruit }\end{array}$ & $\begin{array}{c}\text { Arterial } \\
\text { hypertension }\end{array}$ & S & $\begin{array}{l}\text { HPL } \\
7120 \\
\end{array}$ \\
\hline & Citrus limonum L. & Limão & $\mathrm{Mf}$ & $\mathrm{O}$ & $\operatorname{Tr}$ & $\begin{array}{l}\text { M: cooked with } \\
\text { other foods; } \\
\text { bath leaves } \\
\text { from } O \text {. } \\
\text { campechianum } \\
\text { and Jatropha, } \\
\text { leave in the dew } \\
\text { and wash your } \\
\text { hair the next }\end{array}$ & Flu and cold & S & $\begin{array}{l}\text { MAR } \\
3171\end{array}$ \\
\hline & \multicolumn{9}{|c|}{ Page 23/33 } \\
\hline
\end{tabular}




\begin{tabular}{|c|c|c|c|c|c|c|c|c|c|}
\hline & & & & & & $\begin{array}{l}\text { day / F: use it in } \\
\text { sauces and } \\
\text { broths }\end{array}$ & & & \\
\hline Sapindaceae & $\begin{array}{l}\text { Talisia esculenta (A.St.- } \\
\text { Hil.) Radlk }\end{array}$ & Pitomba & F & Fo & $\mathrm{Sh}$ & Fresh fruit & & $\mathrm{S}$ & $\begin{array}{l}\text { INPA } \\
12607\end{array}$ \\
\hline \multirow[t]{3}{*}{ Sapotaceae } & $\begin{array}{l}\text { Pouteria caimito (Ruiz \& } \\
\text { Pav.) Radlk. }\end{array}$ & Abiu & F & Fo & $\operatorname{Tr}$ & Fresh fruit & & $\mathrm{S}$ & $\begin{array}{l}\text { INPA } \\
10714 \\
\end{array}$ \\
\hline & $\begin{array}{l}\text { Pouteria pachyphylla } \\
\text { Pires }\end{array}$ & Abiurana & F & Fo & $\operatorname{Tr}$ & Fresh fruit & & $\mathrm{S}$ & $\begin{array}{l}\text { INPA } \\
45784\end{array}$ \\
\hline & $\begin{array}{l}\text { Pouteria macrophylla } \\
\text { (Lam.) Eyma }\end{array}$ & Cutite & F & Fo & Sh & Fresh fruit & & $\mathrm{S}$ & $\begin{array}{c}\text { INPA } \\
130432 \\
\end{array}$ \\
\hline Sapotaceae & $\begin{array}{l}\text { Manilkara huberi (Ducke) } \\
\text { Stand. }\end{array}$ & Maçaranduba & Mf & Fo & $\operatorname{Tr}$ & $\begin{array}{l}\text { M: drink the } \\
\text { tree milk / F: } \\
\text { fresh fruit }\end{array}$ & $\begin{array}{l}\text { Improves the } \\
\text { sight }\end{array}$ & $\mathrm{S}$ & $\begin{array}{l}\text { INPA } \\
10320 \\
\end{array}$ \\
\hline \multirow[t]{3}{*}{ Simaroubaceae } & Simarouba amara Aubl. & $\begin{array}{l}\text { Jaruba (aruba, } \\
\text { marupá) }\end{array}$ & $\mathrm{M}$ & Fo & $\operatorname{Tr}$ & Bark tea & Worm & $\mathrm{S}$ & $\begin{array}{l}\text { INPA } \\
10369 \\
\end{array}$ \\
\hline & $\begin{array}{l}\text { Simarouba versicolor A. } \\
\text { St. -Hil. }\end{array}$ & Pau chave & M & Fo & $\operatorname{Tr}$ & $\begin{array}{l}\text { Put tree bark it } \\
\text { in the water, } \\
\text { leave in the } \\
\text { dew, remove } \\
\text { the foam and } \\
\text { drink it } \\
\end{array}$ & Malaria & $\mathrm{S}$ & $\begin{array}{l}\text { INPA } \\
12499\end{array}$ \\
\hline & Quassia amara L. & Quina & $\mathrm{M}$ & Fo & $\operatorname{Tr}$ & $\begin{array}{c}\text { Leaf and bark } \\
\text { tea }\end{array}$ & Malaria & $\mathrm{S}$ & $\begin{array}{l}\text { INPA } \\
4159 \\
\end{array}$ \\
\hline \multirow[t]{4}{*}{ Solanaceae } & Physalis angulata L. & Camapu & Mf & Ff & $\mathrm{He}$ & $\begin{array}{l}\text { Root tea, leaves } \\
\text { from } Q \text {. amara } \\
\text { and } A \text {. } \\
\text { grandifolia, and } \\
\text { L. operculata } \\
\text { fruit dried }\end{array}$ & Malaria & $\mathrm{S}$ & $\begin{array}{c}\text { INPA } \\
106301\end{array}$ \\
\hline & $\begin{array}{l}\text { Solanum sessiliflorum } \\
\text { Dunal }\end{array}$ & Cubiu & $\mathrm{F}$ & Fo & Sh & Fresh fruit & & $\mathrm{S}$ & $\begin{array}{l}\text { INPA } \\
20716 \\
\end{array}$ \\
\hline & Capsicum frutescens L. & $\begin{array}{l}\text { Pimenta } \\
\text { malagueta }\end{array}$ & Mf & $\mathrm{Vg}$ & $\mathrm{He}$ & $\begin{array}{l}\text { M: grind the } \\
\text { leaf with } C \text {. } \\
\text { cyminum and } P \text {. } \\
\text { nigrum seeds / } \\
\text { F: food flavoring }\end{array}$ & Labor pain & $\mathrm{C}$ & $\begin{array}{l}\text { MIRR } \\
5805\end{array}$ \\
\hline & $\begin{array}{l}\text { Solanum americanum } \\
\text { Mill. }\end{array}$ & Pretinha & F & $\mathrm{Rf}$ & $\mathrm{He}$ & Fresh fruit & & $\mathrm{S}$ & $\begin{array}{l}\text { INPA } \\
109121\end{array}$ \\
\hline \multirow[t]{2}{*}{ Talinaceae } & $\begin{array}{l}\text { Talinum paniculatum } \\
\text { (Jacq.) Gaertn. }\end{array}$ & Cariru grande & F & $\begin{array}{l}\mathrm{Vg} \\
\mathrm{Ff}\end{array}$ & $\mathrm{He}$ & $\begin{array}{c}\text { Leaf cooked } \\
\text { with other foods }\end{array}$ & & $\mathrm{C}$ & $\begin{array}{c}\text { INPA } \\
163212 \\
\end{array}$ \\
\hline & $\begin{array}{l}\text { Talinum triangulare } \\
\text { (Jacq.) Willd. }\end{array}$ & $\begin{array}{c}\text { Cariru } \\
\text { pequeno }\end{array}$ & $\mathrm{F}$ & $\begin{array}{l}\mathrm{Vg} \\
\mathrm{Ff}\end{array}$ & $\mathrm{He}$ & $\begin{array}{c}\text { Leaf cooked } \\
\text { with other foods }\end{array}$ & & $\mathrm{C}$ & $\begin{array}{c}\text { INPA } \\
259147 \\
\end{array}$ \\
\hline Urticaceae & $\begin{array}{l}\text { Cecropia pachystachya } \\
\text { Trécul }\end{array}$ & Embaúba & $\mathrm{M}$ & $\mathrm{Rf}$ & $\operatorname{Tr}$ & $\begin{array}{c}\text { Grind the leaf, } \\
\text { put it in the } \\
\text { water and drink } \\
\text { it }\end{array}$ & Diabetes & $\mathrm{S}$ & $\begin{array}{c}\text { INPA } \\
109923\end{array}$ \\
\hline Verbenaceae & Lippia alba (Mill.) N.E. Br. & Carmelitana & Mf & $\mathrm{Vg}$ & $\mathrm{He}$ & $\begin{array}{l}\text { M: leaf tea / F: } \\
\text { leaf cooked with } \\
\text { other foods }\end{array}$ & Headache & $\mathrm{C}$ & $\begin{array}{l}\text { EAFM } \\
12165 \\
\end{array}$ \\
\hline \multirow[t]{2}{*}{ Vitaceae } & $\begin{array}{l}\text { Cissus verticillata (L.) } \\
\text { Nicolson \& C.E. Jarvis }\end{array}$ & Cipó pucá & $\mathrm{M}$ & Fo & $\mathrm{Cl}$ & Bark tea & Stomachache & $\mathrm{S}$ & $\begin{array}{c}\text { INPA } \\
167873 \\
\end{array}$ \\
\hline & Cissus sicyoides L. & Insulina & $\mathrm{M}$ & $\mathrm{Vg}$ & $\mathrm{He}$ & Leaf tea & Diabetes & $\mathrm{C}$ & $\begin{array}{l}\text { EAFM } \\
13214 \\
\end{array}$ \\
\hline \multirow[t]{2}{*}{ Zingiberaceae } & $\begin{array}{l}\text { Zingiber mioga (Thunb.) } \\
\text { Roscoe }\end{array}$ & $\begin{array}{l}\text { Gengibre } \\
\text { grande }\end{array}$ & $\mathrm{Mf}$ & $\begin{array}{l}\mathrm{Vg} \\
\mathrm{O}\end{array}$ & $\mathrm{He}$ & $\begin{array}{c}\text { M: beverage: } H \text {. } \\
\text { courbaril } \\
\text { leaves, } D \text {. } \\
\text { odorata and } B \text {. } \\
\text { orellana seeds, } \\
\text { and honey / } \mathrm{F} \text { : } \\
\text { tea from the } \\
\text { rhizome } \\
\end{array}$ & $\begin{array}{l}\text { Flu, cough and } \\
\text { pneumonia }\end{array}$ & $\mathrm{C}$ & $\begin{array}{l}\text { INPA } \\
5706\end{array}$ \\
\hline & Zingiber officinale Roscoe & $\begin{array}{l}\text { Gengibre } \\
\text { pequena }\end{array}$ & Mf & $\begin{array}{c}\mathrm{Vg}, \\
\mathrm{O}\end{array}$ & $\mathrm{He}$ & $\begin{array}{l}\text { M: beverage: } H . \\
\text { courbaril }\end{array}$ & $\begin{array}{l}\text { Flu, cough and } \\
\text { pneumonia }\end{array}$ & $\mathrm{C}$ & INPA \\
\hline
\end{tabular}




\begin{tabular}{|c|c|c|c|c|c|c|c|c|c|}
\hline & & & & & & $\begin{array}{c}\text { leaves, } D \text {. } \\
\text { odorata and } B \text {. } \\
\text { orellana seeds, } \\
\text { and honey / F: } \\
\text { tea from the } \\
\text { rhizome }\end{array}$ & & & 186157 \\
\hline Not identified & Not identified & $\begin{array}{c}\text { Aririmba } \\
\text { (ariramba) }\end{array}$ & $\mathrm{F}$ & Fo & $\operatorname{Tr}$ & Fresh fruit & & $\mathrm{S}$ & -- \\
\hline Not identified & Not identified & Bolota & $\mathrm{F}$ & Fo & $\mathrm{Sh}$ & Fresh fruit & & $\mathrm{S}$ & -- \\
\hline Not identified & Not identified & $\begin{array}{c}\text { Copaíba de } \\
\text { planta }\end{array}$ & $\mathrm{M}$ & $\mathrm{Vg}$ & $\mathrm{He}$ & $\begin{array}{l}\text { Tea and syrup } \\
\text { of the leaves }\end{array}$ & $\begin{array}{c}\text { Headache and } \\
\text { cough }\end{array}$ & $\mathrm{C}$ & -- \\
\hline Not identified & Not identified & $\begin{array}{c}\text { Cumaru de } \\
\text { planta }\end{array}$ & $\mathrm{M}$ & $\mathrm{Vg}$ & $\mathrm{He}$ & $\begin{array}{c}\text { Syrup of the } \\
\text { leaves }\end{array}$ & Pneumonia & C & -- \\
\hline Not identified & Not identified & $\begin{array}{l}\text { Espinheira } \\
\text { santa }\end{array}$ & $\mathrm{M}$ & Fo & $\operatorname{Tr}$ & $\begin{array}{l}\text { Beverage of the } \\
\text { bark tree with } \\
\text { bark of } S \text {. } \\
\text { adstringens, } U \text {. } \\
\text { tomentosa and } \\
D . \text { subcymosa }\end{array}$ & Gastritis & $\mathrm{S}$ & -- \\
\hline Not identified & Not identified & Japá & $\mathrm{M}$ & Fo & $\operatorname{Tr}$ & $\begin{array}{c}\text { Drink the tree } \\
\text { milk }\end{array}$ & Gastritis & S & - \\
\hline Not identified & Not identified & $\begin{array}{l}\text { Larém } \\
\text { (aralém) }\end{array}$ & M & $\mathrm{Vg}$ & $\mathrm{He}$ & Leaf tea & Malaria & $\mathrm{C}$ & -- \\
\hline Not identified & Not identified & Lua & $\mathrm{F}$ & $\mathrm{Rf}$ & $\mathrm{Cl}$ & Fresh fruit & & $\mathrm{S}$ & -- \\
\hline Not identified & Not identified & Nazarana & $\mathrm{M}$ & Fo & $\operatorname{Tr}$ & $\begin{array}{c}\text { Bath: tree bark, } \\
C \text { deodara bark, } \\
\text { S. guianensis } \\
\text { and } C \text {. cujete } \\
\text { leaves }\end{array}$ & $\begin{array}{c}\text { Fever and "evil } \\
\text { eye" }\end{array}$ & $\mathrm{S}$ & -- \\
\hline Not identified & Not identified & Papagainho & $\mathrm{M}$ & $\mathrm{Vg}$ & $\mathrm{He}$ & Leaf tea & Worm & $\mathrm{C}$ & -- \\
\hline Not identified & Not identified & Pichona & $\mathrm{F}$ & Fo & $\mathrm{Sh}$ & Fresh fruit & & $\mathrm{S}$ & -- \\
\hline Not identified & Not identified & Pracapeá & $\mathrm{F}$ & $\mathrm{Rf}$ & $\operatorname{Tr}$ & Fresh fruit & & $\mathrm{S}$ & -- \\
\hline Not identified & Not identified & Pranari & $\mathrm{F}$ & $\mathrm{Rf}$ & $\mathrm{Tr}$ & Fresh fruit & & $\mathrm{S}$ & -- \\
\hline
\end{tabular}

$($ Cat. $)=$ Category of use, $($ Env. $)=$ Propagation environment, $($ Grow. $)=$ Growth habit, $($ Dom. $)=$ Domestication stage, $($ Veg. $)=$ Vegetative cycle, (Reg.) = Registration of plant species, (M) = Medicinal, (F) = Food, (Mf) = Medicinal and Food, (Vg) = Vegetable garden, (O) = Orchard, (Fo) $=$ Forest, $(\mathrm{Ff})=$ Family farm, $(\mathrm{Rf})$ Riparian forest, $(\mathrm{Tr})=$ Tree, $(\mathrm{Sh})=$ Shrub, $(\mathrm{He})=$ Herbaceous, $(\mathrm{Cr})=\mathrm{Creeper},(\mathrm{Cl})=\mathrm{Climbers},(\mathrm{C})=$ Cultivated, $(\mathrm{S})=$ Spontaneous.

The interviewed reported the existence of some medicinal and food species that are toxic. In this case, they developed some techniques that resulted from the knowledge inherited from their parents. Two examples are cited, the first is the use of $A$. cymbifera, a medicinal species indicated for stomachache and headaches. According to the interviewed, the ingestion of the raw leaf can cause nausea, vomiting and dizziness. In this case, the "poison" is eliminated through the decoction of the leaves. The second example, is the species $S$. americanum, whose fruit is consumed as food, however it is only ingested when it reaches full maturity, defined by the dark color. If consumed before this stage of maturation, it can cause fever, headache and diarrhea.

Regarding the forms of use, particularly for food plant species, it was observed that fruit species are mostly consumed in its fresh form (E. uchi, B. acanthocarpa) and in some cases they are prepared as juices (O. bacaba, O. mapora). Rhizome-producing species are cooked and usually consumed in the breakfast ( $D$. trifida, A. xanthorriza) and vegetables are cooked with other foods ( $E$. foetidum, $H$. sabdariffa).

This survey did not identify the habit of preparing salads with vegetables. However, it was observed that some seeds are used as condiments (B. orellana), paçoca ( $S$. orientale) and consumed in fresh (B. excelsa) and, finally, some of these species are consumed in the form of boiled teas (cooking), during breakfast (C. citratus, C. zeylanicum). 
Regarding the forms of use of medicinal species by the population, there was also a diversity of forms of preparation. They are explained, as it follows:

Beverage: It consists in boiling dry barks of trees and vines. It is indicated for pain, inflammation and different infections in the body. Another way to prepare the beverage is to expose the preparation (water + plant) to the sunlight for 10 to 15 days, until the beverage is completely fermented. The preparation of beverage from medicinal plants is mentioned in several works in the academic literature [51, 53].

Tree bark macerate: Indicated for stomachache, diarrhea and ringworm (topical application). This practice is similar to making teas; however, it is used only with the bark of the trees, and the water is not boiled [35].

Mixture: It is widely used for headache, dizziness, diarrhea, stroke, among other discomforts. It consists of mixing the species indicated for this type of discomfort, fermenting them in alcohol, then putting them in a bottle, and inhaling it. Another form of use for body aches is to massage the sore spot with the mixture.

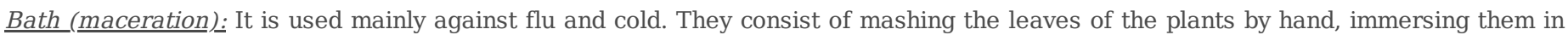
water, and exposing them in dew overnight. The next morning, wash the head with the beverage. Another possibility for preparing the baths: it can be cooked, boil them for approximately one hour, leave it in the dew overnight, and use it the next day [35, 53].

Teas: It is used for medicinal and food purposes, the leaves or peels are immersed in water during boiling. This is the form of use most practiced by rural populations, also known as decoction [51].

Syrup: It is called licker, usually indicated to cure the flu. It is prepared from an oil (C. guianensis), using leaves of some medicinal plant indicated for flu and bee honey. Boil everything together for 30 minutes, then let it cool, and gradually take a spoon three times a day [51, 53].

Juice of the leaves and seeds: Indicated for a particular disease, and ingesting it. It is indicated for symptoms of diarrhea, intestinal parasites and anemia, etc. You can also place it on the site of discomfort when it comes to ringworm, ear pain [43].

Tree milk: It is extracted from the stem of forest species for food and medicinal purposes [54]. In relation to food use, the milk is ingested. In a medical case (ulcer, gastritis, inflammation), it can be ingested or placed over the place where the discomfort occurs (vision problems, toothache, blood stasis, insect bites, muscle strain) [55].

Oil: It is used for food and medicinal purposes. It is used as a condiment in the preparation of food. In medicinal use is ingested fresh and in bottles. The oils are extracted from both the stem of forest species and fruits and seeds [56].

Plant sap: Is collected and consumed immediately after it. It is indicated for problems in the spleen, gastritis, malaise and tiredness [56].

\section{Ethnobotanical indices}

The Shannon-Wiener biological diversity index and the Pielou equitability index were equal to 5.02 and 0.90 , respectively. It was observed the high richness of species of food and medicinal plants in the region under study, and that the knowledge about the use of these species is widely distributed among users of these plant species. This high diversity of plants may be the result of the high ethnobotanical knowledge that traditional, quilombola and indigenous populations develop through a combination of African, Amerindian and European knowledge about plants [33].

Page 26/33 
The species E. foetidum (Apiaceae) and I. batatas (Convolvulaceaea) were the most cited by the interviewed (Table 3) in this study. Also, they obtained a relative citation frequency of 19.70 and 19.33, respectively. The fact that these species are the most cited means that they are the best known [34]. By observing the most cited species (Table 3), it is found that they are species grown close to the households, in domestic gardens or in the fields. They occur spontaneously in the orchards next to the residences. The fact that E. foetidum (Apiaceae), for example, has a dual purpose of use (food and medicine), it can contribute to making it better known and demanded by the interviewed on a daily basis. These species occur significantly in other studies on food and medicinal resources in the Amazon region [43, 57] and other biomes in Brazil [58].

The Use Value Index of the species (Table 3) is ratified by the number of citations and the relative frequency of citations, that is, it is calculated considering the citations of the species by the number of interviewed in the research. The results show how much the species is demanded. The species with the highest relative frequency of citation will also be those with the highest indices of use value, that is, those most demanded by the feeding strategies and local phytotherapy of these interviewed on a daily basis [32].

It is worth noting that the higher the use value of these species, the greater the pressure of use upon them [59, 60]. In the specific case of this study, this analysis is very relevant, especially for the species found in the forest, whose reproduction process is more complex, and for most of them there is still no elaborated agronomic protocol.

Table 3. Relative frequency (Fr) and Value of Use Index (VUI) of the species of food and medicinal plants found in the Cajari River Extractive Reserve, Amazon, Brazil. 


\begin{tabular}{|c|c|c|c|}
\hline Species & Cit. & Fr & IVU \\
\hline E. foetidum & 53 & 19.70 & 0.95 \\
\hline I. batatas & 52 & 19.33 & 0.93 \\
\hline D. trifida & 48 & 17.84 & 0.86 \\
\hline C. guianensis & 47 & 17.47 & 0,84 \\
\hline O. bacaba & 45 & 16.73 & 0.80 \\
\hline C. villosum & 43 & 15.99 & 0.77 \\
\hline D. subcymosa & 42 & 15.61 & 0.75 \\
\hline E. uchi; P. macroloba; T. paniculatum; E. oleracea & 40 & 14.87 & 0.71 \\
\hline C. langsdorfii & 39 & 14.50 & 0.70 \\
\hline A. oleracea; C. cyminum; P. angulata; B. potabile & 38 & 14.13 & 0.68 \\
\hline A. aculeatum & 37 & 13.75 & 0.66 \\
\hline A. camansi; R. graveolens & 36 & 13.38 & 0.64 \\
\hline U. tomentosa; C. anguria; Mentha $x$ villosa & 35 & 13.01 & 0.63 \\
\hline M. flexuosa; C. ambrosioides & 34 & 12.64 & 0.61 \\
\hline P. amboinicus; O campechianum & 33 & 12.27 & 0.59 \\
\hline M. esculenta; C. citratus & 32 & 11.90 & 0.57 \\
\hline S. mombin; I. edulis & 29 & 10.78 & 0.52 \\
\hline J. curcas; A. occidentale; (P. fasciculata & 28 & 10.41 & 0.50 \\
\hline H. courbaril; T. vulgare; M. armata; P. grandis; P. insignis & 27 & 10.04 & 0.48 \\
\hline E. plicata; M. officinalis; S. cumini & 26 & 9.67 & 0.46 \\
\hline Z. mioga; M. grandiflora; A. vera; P. pilosa & 25 & 9.29 & 0.45 \\
\hline C. pepo; Z. officinale; A. esperanzae & 24 & 8.92 & 0.43 \\
\hline X. taioba; A. canelilla; K. brasiliensis; C. limonum; P. barbatus; C. allouia & 23 & 8.55 & 0.41 \\
\hline B. excelsa & 22 & 8.18 & 0.39 \\
\hline Talinum triangulare & 21 & 7.81 & 0.38 \\
\hline H. mariae; F. insipida & 20 & 7.43 & 0.36 \\
\hline Q. amara; C. zeylanicum & 19 & 7.06 & 0.34 \\
\hline P. alliacea & 18 & 6.69 & 0.32 \\
\hline B. rutilans; D. odorata; $M$. alliacea & 17 & 6.32 & 0.30 \\
\hline Papagainho - not identified; T. subincanum & 16 & 5.95 & 0.29 \\
\hline J. gossypiifolia; F. chica; O. basilicum; S. globulifera & 15 & 5.58 & 0.27 \\
\hline V. surinamensis; H. drasticus; L. pisonis; A. esculentus; B. gasipaes; A. muricata; L. alba; J. pectoralis var. stenophylla & 14 & 5.20 & 0.25 \\
\hline P. niruri; P. cablin; P. guajava; Salacia sp.; C. deodara; L. macrophylla; G. hirsutum & 13 & 4.83 & 0.23 \\
\hline C. sinensis; B. alba; C. verticillata & 12 & 4.46 & 0.21 \\
\hline M. citrifolia; E. victoriana; P. macrophylla; S. aromaticum; C. spicatus; S. trifasciata & 11 & 4.09 & 0.20 \\
\hline P. americana & 10 & 3.72 & 0.18 \\
\hline $\begin{array}{l}\text { H. brasiliensis; A. aculeata; P. sericea; B. acanthocarpa; C. papaya; Lua - not identified; E. ayapana; L. operculata; A. } \\
\text { xanthorriza }\end{array}$ & 9 & 3.35 & 016 \\
\hline B. orellana; J. pectoralis; P. nitida; M. indica; Copaíba de planta - not identified; D. dodecaneura & 8 & 2.97 & 0.14 \\
\hline $\begin{array}{l}\text { S. australis; T. esculenta; C. icaco; C. ferrea; S. indicum; T. minuta; O. diandra; C. nucifera; S. adstringens; M. } \\
\text { paradisiaca }\end{array}$ & 7 & 2.60 & 0.13 \\
\hline M. arvensis; M. spicata; S. agrestis; O. bataua; B. crassifolia; P. micropetala; S. orientale; A. vulgaris; A. mucosa & 6 & 2.23 & 0.11 \\
\hline $\begin{array}{l}\text { S. dulcis; T. paniculata; B. pinnatum; A. phalerata; P. grandiflora; P. stratiotes; O. vulgare; G. americana; C. calycularis; } \\
\text { S. terebinthifolia; S. guianensis; A. giganteum; A. tenella }\end{array}$ & 5 & 1.86 & 0.09 \\
\hline J. molissima; P. uncinatum; C. winterianus; P. pellucida; D. bulbifera; M. saccifera; O. mapora; R. officinalis & 4 & 1.49 & 0.07 \\
\hline $\begin{array}{l}\text { H. sabdariffa; A. sorbilis; G. arborescens; C. spruceanum; A. speciosa; P. rohrii; E. triplinerve; I. vulpina; I. sessilis; A. } \\
\text { montana; O. selloi; Cumaru de planta - not identified; C. cujete; A. nitidum; B. caapi; O. coutinhoi; I. batatas; S. } \\
\text { guyanensis; P. pachycarpa; P. caimito }\end{array}$ & 3 & 1.12 & 0.05 \\
\hline $\begin{array}{l}\text { C. rotundus; C. calisaya; C. articulatus; C. frutescens; H. dulcis; S. versicolor; A. murumuru; E. falcata; P. quadrangularis; } \\
\text { L. tomentosa; M. huberi; C. limettiodes; Larém (aralém) - not identified; C. aurantium; V. guianensis; C. brasiliense; M. } \\
\text { maripa; V. faba; S. sessiliflorum; B. exoleta; T. nocturnum; A. carambola; C. americana; E. tirucalli; C. bicolor, Bolota - not } \\
\text { identified; I. purga; G. madruno; P. grandiflora; H. crepitans; A. glabra; H. suaveolens }\end{array}$ & 2 & 0.74 & 0.04 \\
\hline
\end{tabular}

The other species found in the present study were mentioned only once, with Fr and IVU, equal to 0.37 and 0.02 , respectively.

\section{Conclusions}

The study showed that the residents of the Cajari River Extractive Reserve use 269 plant species as food and/or medicinal products, associated with high diversity and equitability. These data reveal the large knowledge about the use of plants in this unit, which

Page 28/33 
constitute a real biocultural heritage of these populations. One of the assets observed in the present study, which differs from others found in this segment, is the interest of the young people in these plants, with a tendency to focus on women and adults and the elderly. This shows the need to register and disseminate the diversity and ways of using these resources, at the risk of losing such knowledge over time. It was also possible to verify the strong relationship of dependence of these populations, since the habitat of most of the species found in the study is the native and riparian forests. Finally, in the last years, the pressure of use on these resources has increased due to the rise in the population, the execution of gardens and fires, which urgently requires the protection, conservation and propagation of many species. These are directly related to the families' survival strategies, and for many of these species, there is still no agronomic protocol that makes it possible to replant them.

\section{Declarations}

\section{Acknowledgments}

The authors are especially grateful to the residents of Cajari River RESEX who participated in this study, for their welcoming and hospitality; the Graduate Program in Phytotechnics at the Federal University of Viçosa (UFV); to the Coordination for the Improvement of Higher Education Personnel (CAPES); to HAMAB taxonomists Patrick Cantuária and Tonny Medeiros for their valuable contributions to species identification.

\section{Authors' contributions}

GXPF and RHSS planned and coordinated the study; GXPF, AFR, AFM and WFP performed the fieldwork; GXPF, WLB and RHSS analyzed the data, discussed the results, wrote and reviewed the manuscript. All authors read and approved the final manuscript.

\section{Funding}

The study has been funded by National Council for Scientific and Technological Development (CNPq) (Edict No. 21/2016), Dean of Research and Graduate Studies at the Federal University of Amapá (PROPESPG/UNIFAP) (Edict No. 14/2017) and by Research Support Foundation of the State of Amapá (FAPEAP) (Edict No. 006/2017).

\section{Availability of data and materials}

The authors already included all data in the manuscript collected during the field surveys. The documented plant species were deposited at Herbarium Collection of Amapá State (HAMAB), Macapá, Amapá, Brazil.

\section{Ethics approval and consent to participate}

During field work, prior consent of the interviewed was taken conducting these studies. This was done to adhere to the ethical standards of community participation in scientific research.

\section{Consent for publication}

Not applicable.

\section{Competing interests}

The authors declare that they have no competing interests. 


\section{Author details}

${ }^{1}$ Departamento de Educação, Universidade Federal do Amapá, Mazagão, Amapá, Brasil. ²Empresa Brasileira de Pesquisa Agropecuária, Macapá, Amapá, Brasil. ${ }^{3}$ Departamento de Agronomia, Universidade Federal de Viçosa, Viçosa, Minas Gerais, Brasil.

\section{References}

1. Metzger JP, Bustamante MMC, Ferreira J, Fernandes GW, Librán-Embid F, Pillar VD, Prist PR, Rodrigues RR, Vieira ICG, Overbeck GE. Why Brazil needs its Legal reserves. PECON. 2019; 17:91-103.

2. Clement CR, Cristo-Araújo M, D’Eeckenbrugge GC, Pereira AA, Picanço-Rodrigues D. Origin and Domestication of Native Amazonian Crops. Divers. 2010; 2:72-106.

3. Newton P, Endo W, Peres CA. Determinants of livelihood strategy variation in two extractive reserves in Amazonian flooded and unflooded forests. Environ Conserv. 2011; 39: 97-110.

4. Silva JB, Simonian LTL. População tradicional, Reservas Extrativistas e racionalidade estatal na Amazônia brasileira. Desenvolv. Meio Ambiente. 2015; 33:163-175.

5. Sinopse do Censo Demográfico de 2010: Instituto Brasileiro de Geografia e Estatística; 2010 [https://censo2010.ibge.gov.br/sinopse/index.php?dados=10\&uf=00].

6. Latrubesse EM, Cozzuol M, Silva-Caminha SAF, Rigsby CA, Absy ML, Jaramillo C. The Late Miocene paleogeography of the Amazon Basin and the evolution of the Amazon River system. Earth Sci Rev. 2010; 99:99-124.

7. Barona E, Ramankutty N, Hyman G, Coomes OT. The role of pasture and soybean in deforestation of the Brazilian Amazon. Environ Res Lett. 2010; 5:1-9.

8. Guedes GR, Brondízio ES, Barbieri AF, Anne R, Penna-Firme R, D’Antona AO. Poverty and Inequality in the Rural Brazilian Amazon: A Multidimensional Approach. Hum Ecol. 2012; 40:41-57.

9. Andrade CS, Rosa LP, Silva NF. Generation of electric energy in isolated rural communities in the Amazon Region a proposal for the autonomy and sustainability of the local populations. Renew Sust Energ Rev. 2011; 15:493-503.

10. Oliveira VB, Yamada LT, Fagg CW, Brandão MGL. Native foods from Brazilian biodiversity as a source of bioactive compounds. Food Res Int. 2012; 48:170-179.

11. Santos JFL, Pagani E, Ramos J, Rodrigues E. Observations on the therapeutic practices of riverine communities of the Unini River, AM, Brazil. J Ethnopharmacol. 2012; 142:503-515.

12. Kinupp VF, Lorenzi H. Plantas Alimentícias Não Convencionais (PANC) no Brasil. São Paulo, Brasil: Instituto Plantarum; 2014.

13. Ahmad K, Pieroni A. Folk knowledge of wild food plants among the tribal communities of Thakhte-Sulaiman Hills, North-West Pakistan. J. Ethnobiol. Ethnomed. 2016; 12:1-15.

14. Leal ML, Alves RP, Hanazaki N. Knowledge, use, and disuse of unconventional food plants. Ethnobiol. Ethnomed. 2018; 14:1-9.

15. Lorenzi H, Matos FJA. Plantas Medicinais no Brasil: nativas e exóticas. São Paulo, Brasil: Instituto Plantarum; 2008.

16. Gras A, Garnatje T, Bonet MÀ, Carrió E, Mayans M, Parada M, Rigat M, Vallès J. Beyond food and medicine, but necessary for life, too: other folk plant uses in several territories of Catalonia and the Balearic Islands. J. Ethnobiol. Ethnomed. 2016; 12:1-53.

17. Couly C, Sist P. Use and knowledge of forest plants among the Ribeirinhos, a traditional Amazonian population. Agroforest Syst. 2013; 87:543:554.

18. Hanazaki N, Herbst DF, Marques MS, Vandebroek I. Evidence of the shifting baseline syndrome in ethnobotanical research. 2013; 9:1-11.

19. Oliveira DR, Kretlli AU, Aguiar ACC, Leitão GG, Vieira MN, Martins KS, et al. Ethnopharmacological evaluation of medicinal plants used against malaria by quilombola communities from Oriximiná, Brazil. J Ethnopharmacol. 2015; 173:424-434.

20. Decreto № 99.145, de 12 de março de 1990. Brasilia: Presidência da República, Casa Civil; 1990 [Available from: http://www.planalto.gov.br/ccivil_03/decreto/1990-1994/D99145.htm].

21. Freitas TLP. A exploração da castanha-do-brasil na resex do Rio Cajari [Tese]. Macapá: Universidade Federal do Amapá; 2013.

22. Etkin NL. Anthropological methods in ethnopharmacology. J Ethnopharmacol. 1993; 38:93-104. 
23. Zank S, Hanazaki N. Exploring the Links between Ethnobotany, Local Therapeutic Practices, and Protected Areas in Santa Catarina Coastline, Brazil. J Evid Based Complementary Altern Med. 2012; 2012 (1-15).

24. Albuquerque UP, Hanazaki N. Recent Developments and Case Studies in Ethnobotany. Recife, Brazil: Brazilian Society of Ethnobiology and Ethnoecology; 2010.

25. Lei № 13.123, de 20 de maio de 2015. Brasília: Presidência da República, Casa Civil; 2015 [Available from: http://www.planalto.gov.br/ccivil_03/_Ato2015-2018/2015/Lei/L13123.htm].

26. Pretty JN, Guijt I, Scoones I, Thompson J. Trainer's Guide for Participatory Learning and action. London: International Institute for Environment and Development; 1995.

27. Albuquerque UP, Lucena RFP. Seleção e escolha dos informantes. In: Albuquerque UP, Lucena RFP, editores. Métodos e técnicas na Pesquisa Etnobotânica. Recife, Brasil: Livro Rápido; 2004.

28. Ming LC. Coleta de plantas medicinais. In: Di Stasi LC, editor. Plantas medicinais: arte e ciência: um guia de estudo interdisciplinar.São Paulo, Brasil: Universidade de São Paulo; 1996.

29. Begossi A. Use of ecological methods in Ethnobotany: diversity index. Econ Bot. 1996; 50(3):280-9.

30. Magurran Ecological diversity and its measurement. London, UK: Croom Helm; 1988.

31. Rossato SC, Leitão-Filho H, Begossi A. An ethnobotany of Caiçáras of the Atlantic Rainforest Coast (Brazil). Econ Bot. 1999; 53(4):387-95.

32. Lucena RFP, Lucena CM, Araújo EL, Alves AGC, Albuquerque UP. Conservation priorities of useful plants from different techniques of collection and analysis of ethnobotanical data. An Acad Bras Ciênc. 2013; 85(1):169-86.

33. Conde BE, Ticktin T, Fonseca AS, Macedo AL, Orsi TO, Chedier LM, et al. Local ecological knowledge and its relationship with biodiversity conservation among two Quilombola groups living in the Atlantic Rainforest, Brazil. Plos One. 2017; 12(11): 1-25.

34. Barreira TF, Paula Filho GX, Rodrigues VCC, Andrade FMC, Santos RHS, Priore SE, et al. Diversidade e equitabilidade de Plantas Alimentícias Não Convencionais na zona rural de Viçosa, Minas Gerais, Brasil. Rev Bras PI Med. 2015; 17(4):964-74.

35. Moreira DL, Guarim-Neto G. Usos múltiplos de plantas do Cerrado: um estudo etnobotânico na comunidade Sítio Pindura, Rosário Oeste, Mato Grosso, Brasil. Polibotánica. 2009; 27: 159-190.

36. Cheikhyoussef A, Shapi M, Matengu K, Ashekele HM. Ethnobotanical study of indigenous knowledge on medicinal plant use by traditional healers in Oshikoto region, Namibia. J Ethnobiol Ethnomed. 2011; 7:1-10.

37. Campos LZO, Albuquerque UP, Peroni N, Araújo EL. Do socioeconomic characteristics explain the knowledge and use of native food plants in semiarid environments in Northeastern Brazil? J Arid Environments. 2015; 115:53-61.

38. Amapá. População: Instituto Brasileiro de Geografia e Estatística; 2018 [Disponível em: https://cidades.ibge.gov.br/brasil/ap/panorama].

39. Pilla MAC, Amorozo MCM. O conhecimento sobre os recursos vegetais alimentares em bairros rurais no Vale do Paraíba, SP, Brasil. Acta Bot Brasilica. 2009; 23:1190-1201.

40. Sujarwo W, Arinasa IBK, Salomone F, Caneva G, Fattorini S. Cultural Erosion of Balinese Indigenous Knowledge of Food and Nutraceutical Plants. Econ Bot. 2014; 68(4):426-437.

41. Bieski IGC, Santos FR, Oliveira RM, Espinosa MM, Macedo M, Albuquerque UP, et al. Ethnopharmacology of Medicinal Plants of the Pantanal Region (Mato Grosso, Brazil). EvidBased Complement Alternat Med. 2012; 49:1-36.

42. Quave CL, Pieroni A. A reservoir of ethnobotanical knowledge informs resilient food security and health strategies in the Balkans. Nat. Plants. 2015; 1:1-6.

43. Vásquez SPF, Mendonça MS, Noda SN. Etnobotânica de plantas medicinais em comunidades ribeirinhas do Município de Manacapuru, Amazonas, Brasil. Acta Amaz. 2014; 44:457-472.

44. Costa JR, Mitja D. $O$ uso dos recursos vegetais por agricultores familiares de Manacapuru (AM). Acta Amaz. 2010; 1:49-58.

45. Silva MS, Fantini AC, Shanley P. Látex de amapá (Parahancornia fasciculata (Poir) Benoist, Apocynaceae): remédio e renda na floresta e na cidade. Bol Mus Para Emílio Goeldi Ciênc Hum. 2011; 6:287-305.

46. Murad W, Ahmad A, Ishaq G, Khan MS, Khan MA, Ullah I, et al. Ethnobotanical studies on plant resources of Hazard Nao Forest, District Malakand, Pakistan. Pak J Weed Sci Res. 2012; 18(4): 509-527.

47. Danikou SJ, Achigan-Dako EG, Wong JLG. Eliciting Local Values of Wild Edible Plants in Southern Bénin to Identify Priority Species for Conservation. Econ Bot. 2011; 65(4):381-395.

Page $31 / 33$ 
48. Fraser JA. Caboclo Horticulture and Amazonian Dark Earths along the Middle Madeira River, Brazil. Hum Ecol. 2010; 38:651-662.

49. Isaac VJ, Almeida MC, Giarrizo T, Deus CP, Vale R, Klein G, et al. Food consumption as an indicator of the conservation of natural resources in riverine communities of the Brazilian Amazon. An Acad Bras Ciênc. 2015; 87(4):2229-2242.

50. Adams C, Murrieta SSS, Sanches RA. Agricultura e alimentação em populações ribeirinhas das várzeas do Amazonas: novas perspectivas. Ambient soc. 2005; 8:1-23.

51. Pilla MAC, Amorozo MCM, Furlan A. Obtenção e uso das plantas medicinais no distrito de Martim Francisco, Município de MogiMirim, SP, Brasil. Acta Bot Brasilica. 2006; 20:789-802.

52. Garlet TMB, Irgang BE. Plantas medicinais utilizadas na medicina popular por mulheres trabalhadoras rurais de Cruz Alta, Rio Grande do Sul, Brasil. Rev Bras PI Med. 2001; 4:9-18.

53. Roque AA, Rocha RM, Loiola MIB. Uso e diversidade de plantas medicinais da Caatinga na comunidade rural de Laginhas, município de Caicó, Rio Grande do Norte (nordeste do Brasil). Rev Bras PI Med. 2010; 12(1):31-42.

54. Bezerra VS, Mattietto RA, Coelho EAA, Aguiar FF. Pasteurização do leite-do-amapá in natura para controle do escurecimento enzimático. Ciênc rural. 2013; 43(9):1715-1720.

55. Pereira ZV, Mussury RM, Almeida AB, Sangalli A. Medicinal plants used by Ponta Porã community, Mato Grosso do Sul State. Acta Sci Biol Sci. 2009; 31(3):293-299.

56. Pasa MC. Saber local e medicina popular: a etnobotânica em Cuiabá, Mato Grosso, Brasil. Bol Mus Para Emílio Goeldi Ciênc Hum. 2011; 6(1):179-196.

57. Leão RBA, Ferreira MRC, Jardim MAG. Levantamento de plantas de uso terapêutico no município de Santa Bárbara do Pará, Estado do Pará, Brasil. 2007; 88(1):21-25.

58. Pasa MC, Soares JJ, Guarm Neto G. Estudo etnobotânico na comunidade de Conceição-Açu (alto da bacia do rio Aricá Açu, MT, Brasil). 2005; 19(2):195-207.

59. Lucena RFP, Soares TC, Vasconcelos Neto CFA, Carvalho TKN, Lucena CM, Alves RRN. Uso de recursos vegetais da Caatinga em uma comunidade rural no Curimataú Paraibano (nordeste do Brasil). Polibotánica. 2012; 34: 237-258.

60. Lucena RFP, Albuquerque UP, Monteiro JM, Almeida CFCBR, Florentino ATN, Ferraz JSF. Useful of the semi-arid northeastern region of Brazil - a look at their conservation and sustainable use. Environ Monit Assess. 2007; 125:281-290.

\section{Figures}




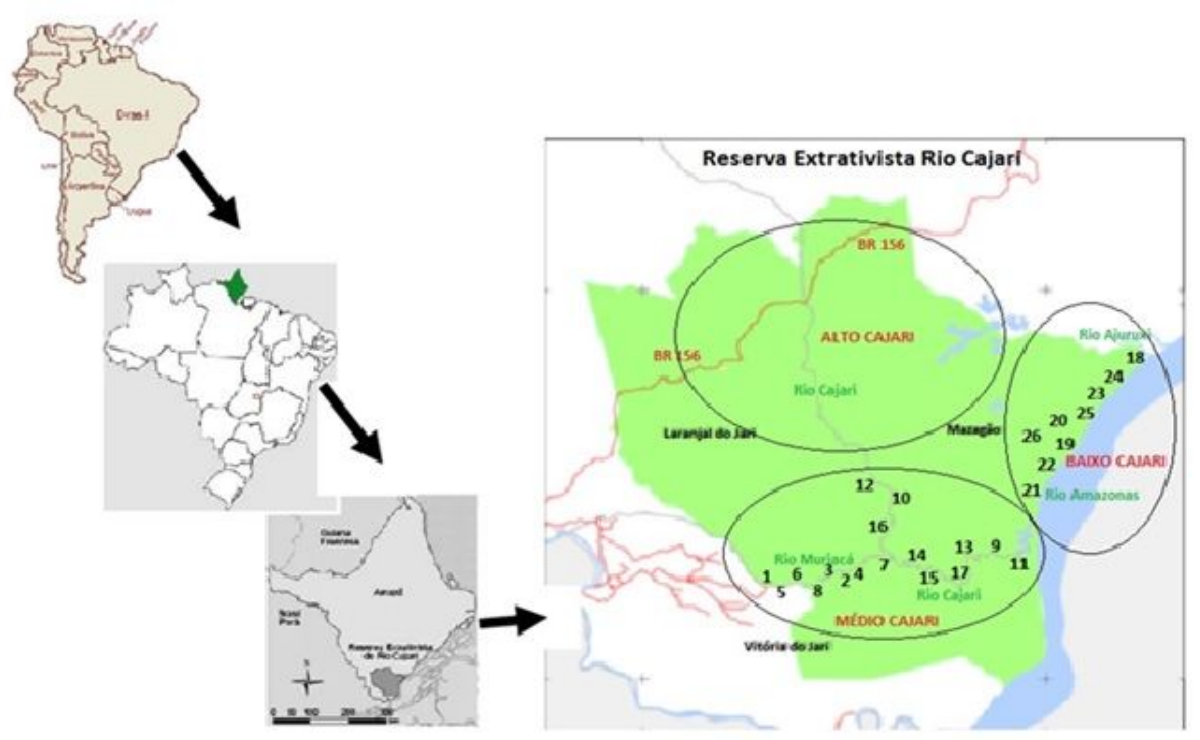

\section{Figure 1}

Study area site, Cajari River Extractive Reserve. Municipalities of Mazagão, Laranjal do Jari and Vitória do Jari, State of Amapá, Brazil. Communities: 1 - Aterro do Muriacá; 2 - Boa Vista; 3 - Comércio; 4 - Conceição do Muriacá; 5 - Mirituba; 6 - Santa Helena; 7 - São Luis; 8 - Vila Nova; 9 - Costureira; 10 - Formigueiro; 11 - Paraíso; 12 - Poção; 13 - Santa Rita; 14 - São Sebastião; 15 - Tapereira; 16 - Terra Vermelha; 17 - Vila Santana; 18 - Foz do Rio Ajuruxi; 19 - Rio Ariramba; 20 - Rio Arirambinha; 21 - Rio Bispo; 22 - Rio Capitão; 23 - Rio Carneiro; 24 - Rio Chato; 25 - Rio Mulato; 26 - Vila Betel. 